\title{
Global Existence and Asymptotic Behavior of Solutions for Nonlinear Wave Equations
}

\section{Kunio Hidano $\mathscr{G}$ Kimitoshi Tsutaya}

\section{Dedicated to Professor K. Kubota on his 60th birthday}

We study the global existence and asymptotic behavior of solutions to semilinear wave equations with two kinds of nonlinear terms. Our main purpose is to show the existence of a scattering operator for small data. The existence of global solutions is proved in the same manner for small initial data given at $t=0$. Our assumptions on the order of nonlinear terms are optimal in view of the nonexistence theorems due to many mathematicians such as F. John. As a consequence, we have succeeded in removing the assumption of spherical symmetry on initial data from the global existence theorem of T. C. Sideris (Comm. P. D. E. 8 (1983), 1291-1323).

1. Introduction. In the present paper we consider a small data scattering problem for semilinear wave equations of the form

$$
\square u=F(D u), \quad t \in \mathbf{R}, \quad x \in \mathbf{R}^{n},
$$

or

$$
\square u=\partial_{t} G(u), \quad t \in \mathbf{R}, \quad x \in \mathbf{R}^{n},
$$

where $\square=\partial_{t}^{2}-\Delta=\partial_{t}^{2}-\sum_{j=1}^{n} \partial_{j}^{2}, \partial_{t}=\partial / \partial t, \partial_{j}=\partial / \partial x_{j}(j=1, \cdots, n), u$ is a real-valued unknown function, $F$ and $G$ represent power nonlinearities of $D u=\left(\partial_{t} u, \nabla u\right)$ and $u$, respectively, satisfying

$$
F(D u)=O\left(|D u|^{\rho}\right) \text { for }|D u| \leq 1
$$

or 


$$
G(u)=O\left(|u|^{\rho}\right) \text { for }|u| \leq 1
$$

with some $\rho>1$. Following the argument in this paper, we could actually study a scattering problem for a wave equation whose nonlinear term is more general than that of (1.2). See Remark 2.2 below. But for simplicity, throughout this paper we will limit ourselves to the nonlinear term $\partial_{t} G(u)$ as in (1.2).

In the scattering theory, the asymptotic behavior of solutions to nonlinear wave equations as $t \rightarrow \pm \infty$ is compared with that of solutions to the free wave equations

$$
\square u=0, \quad t \in \mathbf{R}, \quad x \in \mathbf{R}^{n}
$$

in the energy norm. We first recall the previous results on the Cauchy problem for (1.1) or (1.2). In [11] Lions and Strauss showed by the compactness method that if $F=-\left|\partial_{t} u\right|^{\rho-1} \partial_{t} u$ and $G=-|u|^{\rho-1} u$ with $\rho>1$, then strong solutions to the Cauchy problem for (1.1) and weak solutions to that for (1.2) exist globally in time without any restrictions on the amplitude of data, the order of the nonlinear terms $\rho>1$ and the spatial dimensions $n$. However, when we put no sign conditions on the nonlinear terms, the existence of global solutions can not always be expected. In fact, according to Glassey [3], when smooth data of compact support are large in some sense, the Cauchy problem for (1.1) with $F=\left|\partial_{t} u\right|^{\rho}$ has no global $C^{2}$-solutions for arbitrary $\rho>1$ and $n$. Later, Sideris [15] has extended the result due to Glassey to provide a larger class of examples of $F$, which includes $F=|\nabla u|^{\rho}$ with any $\rho>1$. Moreover, John [8] has shown that when $n=3$ and $F=\left(\partial_{t} u\right)^{2}$, the Cauchy problem for (1.1) never has nontrivial global $C^{3}$-solutions even for small smooth data of compact support. In the same work [8], he also proved that, when $n=3$ and $G=u^{2}$, the Cauchy problem for (1.2) does not have a global $C^{2}$-solution in general even for small smooth data. Therefore, we need to put smallness conditions on data and to restrict the range of $\rho$ in order to discuss the existence of a scattering operator for (1.1) and (1.2). In this paper we show that the scattering operator can be defined for (1.1) in the case $n=2,3$ if $\rho>\rho_{0}(n):=1+2 /(n-1)$. It is also proved that, in the case $n=3$, the scattering operator can be defined for (1.2) if $\rho>\rho_{0}(3)=2$. Namely, let $u_{0}^{-}$be a solution to (1.3) with small data belonging to a suitable Sobolev space. Then we find a global solution $u$ of (1.1) or (1.2) such that

$$
\left\|u(t)-u_{0}^{-}(t)\right\|_{e}:=\frac{1}{\sqrt{2}}\left\{\left\|\partial_{t} u(t)-\partial_{t} u_{0}^{-}(t)\right\|_{L^{2}}^{2}+\left\|u(t)-u_{0}^{-}(t)\right\|_{\dot{H}^{1}}^{2}\right\}^{1 / 2} \rightarrow 0
$$

as $t \rightarrow-\infty$ (the definition of $\dot{H}^{1}$ is given in Section 2). Moreover, there exists a solution $u_{0}^{+}$to (1.3) such that

$$
\left\|u(t)-u_{0}^{+}(t)\right\|_{e} \rightarrow 0 \text { as } t \rightarrow+\infty,
$$

which means that the scattering operator $S:\left(u_{0}^{-}(0), \partial_{t} u_{0}^{-}(0)\right) \mapsto\left(u_{0}^{+}(0), \partial_{t} u_{0}^{+}(0)\right)$ can be defined. In the same manner it is also shown that the Cauchy problems 
for (1.1) with $n=2,3$, and for (1.2) with $n=3$, have global solutions for small initial data if $\rho>\rho_{0}(n)$. As a consequence, we succeed in removing the assumption of spherical symmetry on initial data from Theorem 1 in Sideris [14] (see Remark 6.1 below). We will treat the two-dimensional case of (1.2) separately in a future paper (see Remark 5.1 below).

This lower bound of $\rho$, that is, $\rho_{0}(n)$, is optimal in the following sense. The nonexistence theorems of global solutions for small data have already been established for the Cauchy problem of (1.1) with $F=\left|\partial_{t} u\right|^{\rho}$ or $F=|\nabla u|^{\rho}$ for $1<\rho \leq \rho_{0}(n)(n=2,3)$ (see John [8], Sideris [14] for $n=3$ and Agemi [2], Godin [4], Masuda [12], Schaeffer [13] for $n=2$ ). As is referred to before, John has also proved the nonexistence theorem for $G=u^{2}$ in the case $n=3$ even for small data.

We briefly describe the plan to prove our results. We start with considering the global solvability of the linear problem

$$
\left\{\begin{array}{l}
\square u=h(t, x), \quad t \in \mathbf{R}, x \in \mathbf{R}^{n}, \\
\left\|u(t)-u_{0}^{-}(t)\right\|_{e} \rightarrow 0 \quad(t \rightarrow-\infty)
\end{array}\right.
$$

for given $h$. The solution to (1.4) can be represented formally as

$$
u(t)=u_{0}^{-}(t)+\int_{-\infty}^{t} \frac{\sin \omega(t-\tau)}{\omega} h(\tau) d \tau \quad\left(\omega=(-\Delta)^{1 / 2}\right),
$$

if the right-hand side converges in a suitable norm. However, (1.5) is not useful to the application to our nonlinear problems, because we employ the generalized Sobolev space with weights related to the generators of the Lorentz group (see Section 2). More precisely, since the operators $L_{0}, \Omega_{j k}$ and $L_{j}$ have the weights $x$ and / or $t$, it is not clear whether the operators $L_{0}, \Omega_{j k}$ and $L_{j}$ commute with the integral sign in (1.5). Thus we investigate the approximated solution

$$
u_{\sigma}(t):=u_{0}^{-}(t)+\int_{\sigma}^{t} \frac{\sin \omega(t-\tau)}{\omega} h(\tau) d \tau, \quad \sigma \in(-\infty, 0)
$$

instead of analyzing (1.5) directly. It is shown that the error terms caused by applying $L_{0}$ and $L_{j}$ to (1.6) converge to zero uniformly with respect to $t$ in a suitable norm as $\sigma \rightarrow-\infty$ and therefore we find that Klainerman's method works for the integral form (1.5). We claim that these complicated steps are inevitable when we apply Klainerman's method to the scattering problem, while they are not required in studying the Cauchy problem. For details see Proposition 3.2.

Another difficulty of our problems lies in the lack of smoothness in the nonlinear terms. To show sufficient time decay of the nonlinear terms is important when we intend to construct global solutions in the generalized Sobolev space. If the nonlinear terms were sufficiently smooth, then Klainerman's inequality (see 
(3.7)) would be enough to show good time decay of the nonlinear terms. However, since the nonlinear terms in (1.1) and (1.2) are not so smooth, we have to make effective use of the $L^{p}-L^{q}$ inequality (see (3.9)) in addition to Klainerman's one in order to construct global solutions in the generalized Sobolev space with low regularity. Finally, when constructing solutions to (1.1) or (1.2), we apply a variant contraction mapping theorem instead of the usual iteration as in John [7] because of the lack of smoothness in the nonlinear terms.

The lack of smoothness in the nonlinear terms also causes it difficult to study a small data scattering problem for (1.1) or (1.2) with $\rho>\rho_{0}(n)$ in higher dimensional space.

This paper is organized as follows. In the next section we give several notations and then state our theorems concerning the existence of a scattering operator for (1.1) and (1.2). In Section 3 we prove some results frequently used in Sections 4 and 5, where the proofs of Theorems 2.1 and 2.2 are carried out. In the last section our argument is applied to the Cauchy problems related to (1.1) and (1.2).

2. Notations and theorems. Following Klainerman [9], [10], we introduce several partial differential operators as follows: $D_{0}=\partial_{t}, D_{1}=\partial_{1}, \ldots, D_{n}=\partial_{n}$, $L_{j}=t \partial_{j}+x_{j} \partial_{t}(j=1, \ldots, n), \Omega_{j k}=x_{j} \partial_{k}-x_{k} \partial_{j}(1 \leq j<k \leq n), L_{0}=$ $t \partial_{t}+x_{1} \partial_{1}+\cdots+x_{n} \partial_{n}$. These operators $D_{0}, \ldots, D_{n}, L_{1}, \ldots, L_{n}, \Omega_{12}, \ldots, \Omega_{n-1 n}$, $L_{0}$ are denoted by $\Gamma_{0}, \ldots, \Gamma_{\mu}$ in this order, where $\mu=\left(n^{2}+3 n+2\right) / 2$. Let $N$ be a non-negative integer. We define the norm

$$
\|u(t)\|_{N, p}:=\sum_{|\alpha| \leq N}\left(\int_{\mathbf{R}^{n}}\left|\Gamma^{\alpha} u(t, x)\right|^{p} d x\right)^{1 / p} \quad(1 \leq p<\infty)
$$

for any function $u(t, x)$ for which the right-hand side above is finite for every $t \in \mathbf{R}$. Here $\alpha$ is a multi-index, $|\alpha|=\alpha_{0}+\cdots+\alpha_{\mu}$ and $\Gamma^{\alpha}=\Gamma_{0}^{\alpha_{0}} \cdots \Gamma_{\mu}^{\alpha_{\mu}}$. We also define the norm

$$
\|D u(t)\|_{N, p}:=\sum_{k=0}^{n}\left\|D_{k} u(t)\right\|_{N, p} \quad(1 \leq p<\infty)
$$

for a vector $D u$. In particular, $\|u(t)\|_{0, p}$ and $\|D u(t)\|_{0, p}$ are simply denoted by $\|u(t)\|_{p}$ and $\|D u(t)\|_{p}$, respectively. For a non-negative integer $s, W^{s, p}\left(\mathbf{R}^{n}\right)$ means the usual Sobolev space on $\mathbf{R}^{n}$ with the norm $\|v\|_{W^{s, p}}$. Especially, we put $H^{s}\left(\mathbf{R}^{n}\right)=W^{s, 2}\left(\mathbf{R}^{n}\right), L^{2}\left(\mathbf{R}^{n}\right)=W^{0,2}\left(\mathbf{R}^{n}\right)$. We denote by $\mathcal{S}\left(\mathbf{R}^{n}\right)$ the space of Schwartz's rapidly decreasing functions. Let $\hat{v}$ or $\mathcal{F}[v]$ mean the Fourier transform of $v \in \mathcal{S}\left(\mathbf{R}^{n}\right)$ :

$$
\hat{v}(\xi)=\mathcal{F}[v](\xi)=\int_{\mathbf{R}^{n}} v(x) \exp [-i x \cdot \xi] d x \quad(i=\sqrt{-1}) .
$$


We denote by $\mathcal{F}^{-1}$ the inverse Fourier transform. We also define the Fourier transform for $v \in \mathcal{S}^{\prime}\left(\mathbf{R}^{n}\right)$ (tempered distribution) in the usual way and denote it by $\hat{v}$ or $\mathcal{F}[v]$ likewise. $\mathcal{F}^{-1}$ means the inverse Fourier transform. Set $w(x)=$ $\sqrt{1+|x|^{2}}$. For non-negative integers $s$ and $m$ let $\dot{H}^{s}\left(\mathbf{R}^{n}\right)$ and $\dot{H}^{s, m}\left(\mathbf{R}^{n}\right)$ be the Banach spaces defined by the completion of $\mathcal{S}\left(\mathbf{R}^{n}\right)$ with respect to the seminorms

$$
\begin{aligned}
& \|v\|_{\dot{H}^{s}}=\sum_{|\gamma|=s}\left\|D_{x}^{\gamma} v\right\|_{L^{2}}, \\
& \|v\|_{\dot{H}^{s, m}}=\sum_{|\gamma|=s}\left\|w^{m} D_{x}^{\gamma} v\right\|_{L^{2}},
\end{aligned}
$$

respectively. Here $\gamma$ is a multi-index with $\gamma=\left(\gamma_{1}, \ldots, \gamma_{n}\right), D_{x}^{\gamma}=\partial_{1}^{\gamma_{1}} \cdots \partial_{n}^{\gamma_{n}}$. In the case of $n=3$ we define the Banach space $\dot{H}^{-1}\left(\mathbf{R}^{3}\right)$ by the completion of $\mathcal{S}\left(\mathbf{R}^{3}\right)$ with respect to the seminorm $\|v\|_{\dot{H}^{-1}}=\left\|\mathcal{F}^{-1}\left[|\xi|^{-1} \hat{v}\right]\right\|_{L^{2}}$. We denote by $\|u(t)\|_{e}$ the energy norm

$$
\|u(t)\|_{e}=\frac{1}{\sqrt{2}}\left\{\left\|\partial_{t} u(t)\right\|_{2}^{2}+\|u(t)\|_{\dot{H}^{1}}^{2}\right\}^{1 / 2} .
$$

For any slowly increasing function $H=H(|\xi|)$ in $\mathbf{R}_{\xi}^{n}$ we define the operator $H\left((-\Delta)^{1 / 2}\right)$ as

$$
H\left((-\Delta)^{1 / 2}\right) v=\mathcal{F}^{-1}[H(|\xi|) \hat{v}]
$$

for $v \in \mathcal{S}^{\prime}\left(\mathbf{R}^{n}\right) . H\left((-\Delta)^{1 / 2}\right)$ maps $\mathcal{S}^{\prime}\left(\mathbf{R}^{n}\right)$ into itself. We put $\omega=(-\Delta)^{1 / 2}$ for simplicity. (For the definition of slowly increasing functions, see, e.g., Yoshida [16] on page 150.)

We next state the assumptions on $F$ and $G$. Let $\lambda=\left(\lambda_{0}, \ldots, \lambda_{n}\right) \in \mathbf{R}^{n+1}$ with $|\lambda|=\sqrt{\lambda_{0}^{2}+\cdots+\lambda_{n}^{2}}$ and let $\beta=\left(\beta_{0}, \ldots, \beta_{n}\right)$ be a multi-index with $|\beta|=$ $\beta_{0}+\cdots+\beta_{n}$. We put the following assumptions on $F$ :

(F.1) $F(\lambda) \in C^{2}\left(\mathbf{R}^{n+1}\right)$.

(F.2) There exist some $\rho>\rho_{0}(n)$ and $A_{1}>0$ such that

$$
\left|\partial_{\lambda}^{\beta} F(\lambda)\right| \leq A_{1}|\lambda|^{\rho-|\beta|}(|\beta| \leq 2) \text { for }|\lambda| \leq 1 \text {, where } \partial_{\lambda}^{\beta}=\partial_{\lambda_{0}}^{\beta_{0}} \cdots \partial_{\lambda_{n}}^{\beta_{n}} .
$$

(F.3) For $|\beta|=2$ there exists some $A_{2}>0$ such that

$$
\left|\partial_{\lambda}^{\beta} F(\lambda)-\partial_{\lambda}^{\beta} F(\tilde{\lambda})\right| \leq A_{2}(|\lambda|+|\tilde{\lambda}|)^{\nu_{0}}|\lambda-\tilde{\lambda}|^{\nu_{1}} \text { for }|\lambda|,|\tilde{\lambda}| \leq 1 / 2,
$$

where $\left(\nu_{0}, \nu_{1}\right)=(\rho-3,1)$ if $n=2$ and

$$
\left(\nu_{0}, \nu_{1}\right)= \begin{cases}(0, \rho-2) & \text { if } 2<\rho<3 \\ (\rho-3,1) & \text { if } \rho \geq 3\end{cases}
$$

if $n=3$. 
We put the assumptions on $G$ as follows:

(G.1) $G(z) \in C^{2}(\mathbf{R})$.

(G.2) There exist some $\rho>\rho_{0}(3)=2$ and $A_{3}>0$ such that

$$
\left|d^{k} G(z) / d z^{k}\right| \leq A_{3}|z|^{\rho-k}(k \leq 2) \text { for }|z| \leq 1 .
$$

(G.3) There exists some $A_{4}>0$ such that

$$
\left|G^{\prime \prime}(z)-G^{\prime \prime}(\tilde{z})\right| \leq A_{4}(|z|+|\tilde{z}|)^{\nu_{0}}|z-\tilde{z}|^{\nu_{1}} \text { for }|z|,|\tilde{z}| \leq 1 / 2,
$$

where ${ }^{\prime \prime}=d^{2} / d z^{2}$ and $\left(\nu_{0}, \nu_{1}\right)$ is the same as in (F.3) with $n=3$.

Example. A typical example satisfying (F.1)-(F.3) is $\pm\left|\partial_{t} u\right|^{\rho-1} \partial_{t} u$ or $\pm\left|\partial_{t} u\right|^{\rho}$ with $\rho>\rho_{0}(n)$. For $G(u), \pm|u|^{\rho-1} u$ or $\pm|u|^{\rho}$ with $\rho>\rho_{0}(3)$ satisfies (G.1)-(G.3).

Now we can state our theorems.

Theorem 2.1. Let $n=2,3$. Suppose that (F.1)-(F.3) hold and that $f \in$ $\bigcap_{k=0}^{2} \dot{H}^{1+k, k}\left(\mathbf{R}^{n}\right), g \in \bigcap_{k=0}^{2} \dot{H}^{k, k}\left(\mathbf{R}^{n}\right)$. Let $u_{0}^{-}$be a solution to (1.3) with $\left(u_{0}^{-}(0), \partial_{t} u_{0}^{-}(0)\right)=(f, g)$.

(i) Then there exists a positive constant $\delta_{0}$ depending on $n, \rho, A_{1}$, and $A_{2}$ such that if

$$
\delta:=\sum_{k=0}^{2}\|f\|_{\dot{H}^{1+k, k}}+\sum_{k=0}^{2}\|g\|_{\dot{H}^{k, k}} \leq \delta_{0},
$$

then the equation (1.1) has a unique solution $u=u(t, x)$ satisfying

(2.2) $u \in C\left(\mathbf{R} ; \dot{H}^{1}\left(\mathbf{R}^{n}\right) \cap \dot{H}^{3}\left(\mathbf{R}^{n}\right)\right)$,

(2.3) $\partial_{t} u \in \bigcap_{k=0}^{2} C^{k}\left(\mathbf{R} ; H^{2-k}\left(\mathbf{R}^{n}\right)\right)$,

(2.4) $\Gamma^{\alpha} D_{k} u \in C\left(\mathbf{R} ; L^{2}\left(\mathbf{R}^{n}\right)\right)$ for any $\alpha$ with $|\alpha|=1,2$ and $k=0, \cdots, n$,

(2.5) $\sup _{t \in \mathbf{R}}\|D u(t)\|_{2,2} \leq 2 C_{1} \delta$ with some constant $C_{1}>0$,

(2.6) $\left\|D\left\{u(t)-u_{0}^{-}(t)\right\}\right\|_{2,2}=O\left(|t|^{-(n-1)(\rho-1) / 2+1}\right)$ as $t \rightarrow-\infty$.

(ii) Moreover, there exists a unique solution $u_{0}^{+}=u_{0}^{+}(t, x)$ to (1.3) satisfying

$$
\begin{aligned}
& \text { (2.7) } u_{0}^{+} \in C\left(\mathbf{R} ; \dot{H}^{1}\left(\mathbf{R}^{n}\right) \cap \dot{H}^{3}\left(\mathbf{R}^{n}\right)\right), \\
& (2.8) \quad \partial_{t} u_{0}^{+} \in \bigcap_{k=0}^{2} C^{k}\left(\mathbf{R} ; H^{2-k}\left(\mathbf{R}^{n}\right)\right), \\
& (2.9) \quad \Gamma^{\alpha} D_{k} u_{0}^{+} \in C\left(\mathbf{R} ; L^{2}\left(\mathbf{R}^{n}\right)\right) \text { for any } \alpha \text { with }|\alpha|=1,2 \text { and } k= \\
& 0, \cdots, n,
\end{aligned}
$$$$
\text { (2.10) } \sup _{t \in \mathbf{R}}\left\|D u_{0}^{+}(t)\right\|_{2,2} \leq 2 C_{1} \delta,
$$ 


$$
\left\|D\left\{u(t)-u_{0}^{+}(t)\right\}\right\|_{2,2}=O\left(t^{-(n-1)(\rho-1) / 2+1}\right) \text { as } t \rightarrow+\infty .
$$

Thus the scattering operator

$$
S:\left(u_{0}^{-}(0), \partial_{t} u_{0}^{-}(0)\right) \mapsto\left(u_{0}^{+}(0), \partial_{t} u_{0}^{+}(0)\right)
$$

can be defined for (1.1).

Another theorem is concerned with the existence of a scattering operator for (1.2).

Theorem 2.2. Let $n=3$. Suppose that (G.1)-(G.3) hold and that $f \in$ $\bigcap_{k=0}^{2} \dot{H}^{k, k}\left(\mathbf{R}^{3}\right), g \in \bigcap_{k=0}^{1} \dot{H}^{k, k+1}\left(\mathbf{R}^{3}\right)$. Let $u^{-}$be a solution to (1.3) with $\left(u^{-}(0), \partial_{t} u^{-}(0)\right)=(f, g)$.

(i) Then there exists a positive constant $\eta_{0}$ depending on $\rho, A_{3}$ and $A_{4}$ such that if

$$
\eta:=\sum_{k=0}^{2}\|f\|_{\dot{H}^{k, k}}+\sum_{k=0}^{1}\|g\|_{\dot{H}^{k, k+1}} \leq \eta_{0},
$$

then the equation (1.2) has a unique solution $u=u(t, x)$ satisfying

$$
\begin{aligned}
& u \in \bigcap_{k=0}^{2} C^{k}\left(\mathbf{R} ; H^{2-k}\left(\mathbf{R}^{3}\right)\right), \\
& \Gamma^{\alpha} u \in C\left(\mathbf{R} ; L^{2}\left(\mathbf{R}^{3}\right)\right) \text { for any } \alpha \text { with }|\alpha| \leq 2, \\
& \sup _{t \in \mathbf{R}}\|u(t)\|_{2,2} \leq 2 C_{2} \eta \text { with some constant } C_{2}>0 \\
& \left\|u(t)-u^{-}(t)\right\|_{2,2}=O\left(|t|^{-\rho+2}\right) \text { as } t \rightarrow-\infty
\end{aligned}
$$

(ii) Moreover, there exists a unique solution $u^{+}=u^{+}(t, x)$ to (1.3) satisfying

$$
\begin{aligned}
& u^{+} \in \bigcap_{k=0}^{2} C^{k}\left(\mathbf{R} ; H^{2-k}\left(\mathbf{R}^{3}\right)\right), \\
& \Gamma^{\alpha} u^{+} \in C\left(\mathbf{R} ; L^{2}\left(\mathbf{R}^{3}\right)\right) \text { for any } \alpha \text { with }|\alpha| \leq 2, \\
& \sup _{t \in \mathbf{R}}\left\|u^{+}(t)\right\|_{2,2} \leq 2 C_{2} \eta, \\
& \left\|u(t)-u^{+}(t)\right\|_{2,2}=O\left(t^{-\rho+2}\right) \text { as } t \rightarrow+\infty .
\end{aligned}
$$

Thus the scattering operator

$$
S:\left(u^{-}(0), \partial_{t} u^{-}(0)\right) \mapsto\left(u^{+}(0), \partial_{t} u^{+}(0)\right)
$$

can be defined for (1.2).

Remark 2.1. When $n \geq 3$, the condition of $w v \in L^{2}\left(\mathbf{R}^{n}\right)$ implies $v \in$ $\dot{H}^{-1}\left(\mathbf{R}^{n}\right)$ and $\|v\|_{\dot{H}^{-1}} \leq C\|w v\|_{L^{2}}$. 
Remark 2.2. All the conclusions of Theorem 2.2 can be obtained for the wave equations

$$
\square u=\sum_{j=0}^{3} a_{j} \partial_{j}\left(|u|^{\rho_{j}}\right)+\sum_{j=0}^{3} b_{j} \partial_{j}\left(|u|^{\tilde{\rho}_{j}-1} u\right) \text { in } \mathbf{R} \times \mathbf{R}^{3}
$$

with $a_{j}, b_{j} \in \mathbf{R}, \rho_{j}, \tilde{\rho}_{j}>2(0 \leq j \leq 3), \partial_{0}:=\partial_{t}$ if we replace $\eta_{0}$ and $\rho$ in (2.16), (2.20) by a suitable $\tilde{\eta}_{0}>0$ and $\tilde{\rho}:=\min \left\{\rho_{j}, \tilde{\rho}_{j} \mid j=0, \ldots, 3\right\}$ respectively. Its proof is the same as that of Theorem 2.2.

3. Preliminary results. In this section we prepare several lemmas and propositions which will be frequently used in the proofs of Theorems 2.1 and 2.2. In what follows different constants will be denoted by $C$. Let $[\cdot, \cdot]$ be a commutator and $\delta_{j k}$ be the Kronecker delta. Then we have the commutation relations as follows.

Lemma 3.1. The following equalities

$$
\begin{aligned}
& {\left[\Gamma_{j}, \Gamma_{k}\right]=\sum_{m=0}^{\mu} C_{j k m} \Gamma_{m},} \\
& {\left[\Gamma_{k}, D_{\ell}\right]=\sum_{m=0}^{n} \widetilde{C}_{k \ell m} D_{m}}
\end{aligned}
$$

hold for $j, k=0, \ldots, \mu, \ell=0, \ldots, n$ with coefficients $C_{j k m}, \widetilde{C}_{k \ell m} \in\{-1,0,1\}$. In particular,

$$
\begin{aligned}
& {\left[L_{j}, \partial_{k}\right]=-\delta_{j k} \partial_{t} \text { for } j, k=1, \ldots, n,} \\
& {\left[\Omega_{j k}, \partial_{\ell}\right]=-\delta_{j \ell} \partial_{k}+\delta_{k \ell} \partial_{j} \text { for } 1 \leq j<k \leq n, \ell=1, \ldots, n,} \\
& {\left[L_{0}, \partial_{k}\right]=-\partial_{k} \text { for } k=1, \ldots, n}
\end{aligned}
$$

This lemma is easily verified by direct calculations. Thus we omit the proof.

Let $*$ mean the spatial convolution and set $f_{n \nu}(x):=|x|^{-n+\nu}$. Put $w(x)=$ $\sqrt{1+|x|^{2}}$ as before. The following proposition is useful to derive the time decay estimate for the nonlinear terms. 
Proposition 3.2. For any function $u=u(t, x)$ for which the norm appearing on the right-hand side below is finite for every $t \in \mathbf{R}$ it holds that

$$
\begin{aligned}
& |u(t, x)| \leq C\|u(t)\|_{H^{s}}, s>n / 2, \\
& \|u(t)\|_{q} \leq C\|u(t)\|_{W^{s, p}}, 0<s \leq n / p, 1 \leq p \leq q<\infty, 1 / q \geq 1 / p-s / n, \\
& |u(t, x)| \leq C(1+|t|+|x|)^{-(n-1) / 2}(1+\|t|-| x\|)^{-1 / 2}\|u(t)\|_{s, 2}, s>n / 2, \\
& \left\|w^{(n-1)(1 / p-1 / q)} u(t)\right\|_{q} \\
& \leq C \sum_{|\gamma|+|\theta| \leq s}\left\|D_{x}^{\gamma} \Omega^{\theta} u(t)\right\|_{p}, 0<s \leq n / p, 1 \leq p \leq q<\infty, 1 / q \geq 1 / p-s / n, \\
& \|u(t)\|_{q} \leq C(1+|t|)^{-(n-1)(1 / p-1 / q)}\|u(t)\|_{s, p}, \\
& \quad 0<s \leq n / p, 1 \leq p \leq q<\infty, 1 / q \geq 1 / p-s / n, \\
& \left\|f_{n \nu} * u(t)\right\|_{q} \quad C\|u(t)\|_{p}, 1<p<q<\infty, 1 / q=1 / p-\nu / n .
\end{aligned}
$$

Here $\gamma$ and $\theta$ are multi-indices with $\gamma=\left(\gamma_{1}, \cdots, \gamma_{n}\right), \theta=\left(\theta_{12}, \cdots, \theta_{n-1 n}\right)$, $|\gamma|=\gamma_{1}+\cdots+\gamma_{n},|\theta|=\theta_{12}+\cdots+\theta_{n-1 n}, D_{x}^{\gamma}=\partial_{1}^{\gamma_{1}} \cdots \partial_{n}^{\gamma_{n}}, \Omega^{\theta}=\prod_{1 \leq j<k \leq n} \Omega_{j k}^{\theta_{j k}}$.

Proof. The inequalities (3.5) and (3.6) are well-known (see, e.g., Adams [1] on page 97$)$. Inequality (3.7) is a special case of the Klainerman's one in [10]. The proof of (3.8) can be found in the section 3 of Hörmander [6]. Inequality (3.10) is the Hardy-Littlewood-Sobolev inequality (see, e.g., Hörmander [5] on page 117). Thus we have only to show (3.9). It is enough to show (3.9) for smooth functions. We start with the usual Sobolev inequality on the unit ball $B_{1}^{y}:=\left\{y\left|y \in \mathbf{R}^{n},\right| y \mid \leq 1\right\}:$

$$
\begin{aligned}
& \|v(t)\|_{L^{q}\left(B_{1}^{y}\right)} \leq C\|v(t)\|_{W^{s, p}\left(B_{1}^{y}\right)}, \\
& \quad 0<s \leq n / p, 1 \leq p \leq q<\infty, 1 / q \geq 1 / p-s / n .
\end{aligned}
$$

Then, making the change of variables $x=\zeta y$ for $\zeta>0$, we see by a simple scaling argument that

$$
\begin{aligned}
& \|u(t)\|_{L^{q}\left(B_{\zeta}\right)} \leq C \zeta^{-n(1 / p-1 / q)} \sum_{|\gamma| \leq s} \zeta^{|\gamma|}\left\|D_{x}^{\gamma} u(t)\right\|_{L^{p}\left(B_{\zeta}\right)} \\
& \\
& \text { for } u(t, x):=v\left(t, \zeta^{-1} x\right),
\end{aligned}
$$

if $0<s \leq n / p, 1 \leq p \leq q<\infty, 1 / q \geq 1 / p-s / n$. Here and below $B_{\zeta}$ means $\left\{x\left|x \in \mathbf{R}^{n},\right| x \mid \leq \zeta\right\}$. As has been noted in Klainerman [10] (see [10] on page 115), the inequality

$$
\left|\partial_{j} u(t, x)\right| \leq C|| t|-| x||^{-1}|\Lambda u(t, x)|
$$


holds because

$$
\partial_{j}=\frac{t L_{j}+\sum_{k=1}^{n} x_{k}\left(x_{j} \partial_{k}-x_{k} \partial_{j}\right)-x_{j} L_{0}}{t^{2}-|x|^{2}} \quad(j=1, \ldots, n) .
$$

Here we have set $\Lambda u=\left(L_{j} u, \Omega_{k \ell} u, L_{0} u\right)(j=1, \ldots, n, 1 \leq k<\ell \leq n)$. Moreover, since $L_{j} \cdot\left(t^{2}-|x|^{2}\right)^{-1}=\Omega_{k \ell} \cdot\left(t^{2}-|x|^{2}\right)^{-1}=0$ and $L_{0} \cdot\left(t^{2}-|x|^{2}\right)^{-1}=$ $-2\left(t^{2}-|x|^{2}\right)^{-1}$, a simple observation shows that

$$
\left|D_{x}^{\gamma} u(t, x)\right| \leq\left. C|| t|-| x\right|^{-|\gamma|}\left|\Lambda^{\gamma} u(t, x)\right| \quad \text { for every } \gamma,
$$

where $\Lambda^{\gamma} u$ represents the vector formed by all $\Lambda_{1} \cdots \Lambda_{m} u(1 \leq m \leq|\gamma|)$ with $\Lambda_{1}, \ldots, \Lambda_{m}$ any of the operators $L_{j}, \Omega_{k \ell}$ and $L_{0}$. This inequality implies that

$$
\left|D_{x}^{\gamma} u(t, x)\right| \leq C(1+|| t|-| x||)^{-|\gamma|}\left(\left|D_{x}^{\gamma} u(t, x)\right|+\left|\Lambda^{\gamma} u(t, x)\right|\right) .
$$

Put $\zeta=(1+|t|) / 2$. Since $1+|| t|-| x|| \geq(1+|t|) / 2$ on $B_{(1+|t|) / 2}$, we obtain from (3.11) and (3.12)

$$
\begin{aligned}
\|u(t)\|_{L^{q}\left(B_{(1+|t|) / 2}\right)} & \leq C(1+|t|)^{-n(1 / p-1 / q)}\|u(t)\|_{s, p}, \\
& 0<s \leq n / p, 1 \leq p \leq q<\infty, 1 / q \geq 1 / p-s / n .
\end{aligned}
$$

On the other hand, it follows from (3.8) that

$$
\begin{array}{r}
(1+|t|)^{(n-1)(1 / p-1 / q)}\|u(t)\|_{L^{q}\left(\mathbf{R}^{n} \backslash B_{(1+|t|) / 2}\right)} \leq C \sum_{|\gamma|+|\theta| \leq s}\left\|D_{x}^{\gamma} \Omega^{\theta} u(t)\right\|_{p}, \\
0<s \leq n / p, 1 \leq p \leq q<\infty, 1 / q \geq 1 / p-s / n .
\end{array}
$$

Combining (3.13) and (3.14), we have proved (3.9).

Remark 3.1. The positive constant $C=C_{n, s}$ on the right-hand side of (3.5) depends on $n$ and $s$. We put $C_{3}=\max \left\{C_{2,2}, C_{3,2}\right\}$ for the argument in what follows.

Consider the global solvability of the linear problem

$$
\begin{aligned}
& \square u=h(t, x), \quad t \in \mathbf{R}, x \in \mathbf{R}^{n}, \\
& \left\|u(t)-u_{0}^{-}(t)\right\|_{e} \rightarrow 0 \quad(t \rightarrow-\infty),
\end{aligned}
$$

where $n=2,3$ and $u_{0}^{-}$is a solution to $(1.3)$ with $\left(u_{0}^{-}(0), \partial_{t} u_{0}^{-}(0)\right)=(f, g)$. Let $\rho>\rho_{0}(n)(n=2,3)$. We put the assumptions on $h$ as follows:

$$
\begin{aligned}
& \Gamma^{\alpha} h \in C\left(\mathbf{R} ; L^{2}\left(\mathbf{R}^{n}\right)\right), \\
& \left\||x| \Gamma^{\alpha} h(t)\right\|_{2} \leq C(1+|t|)^{-(n-1)(\rho-1) / 2+1}
\end{aligned}
$$


for any $\alpha$ with $|\alpha| \leq 1$ and

$$
\|h(t)\|_{2,2} \leq C(1+|t|)^{-(n-1)(\rho-1) / 2} .
$$

Set

$$
\begin{aligned}
I_{\sigma}(t) & :=\int_{\sigma}^{t} \frac{\sin \omega(t-\tau)}{\omega} h(\tau) d \tau \\
J_{\sigma}(t) & :=\int_{\sigma}^{t} \cos \omega(t-\tau) \cdot h(\tau) d \tau
\end{aligned}
$$

for any $\sigma \in \mathbf{R}$. Then we obtain the following result.

Lemma 3.2. Assume (3.17)-(3.19). Then the following equalities hold:

$$
\begin{aligned}
L_{j} \partial_{t} I_{\sigma}(t)= & L_{j} J_{\sigma}(t) \\
= & \cos \omega(t-\sigma) \cdot\left(x_{j} h(\sigma)\right)-\int_{\sigma}^{t} \frac{\sin \omega(t-\tau)}{\omega} \partial_{j} h(\tau) d \tau \\
& \quad+\int_{\sigma}^{t} \cos \omega(t-\tau) \cdot L_{j} h(\tau) d \tau \quad(j=1, \ldots, n),
\end{aligned}
$$

$$
\begin{array}{rlr}
\Omega_{j k} \partial_{t} I_{\sigma}(t) & =\Omega_{j k} J_{\sigma}(t) \\
& =\int_{\sigma}^{t} \cos \omega(t-\tau) \cdot \Omega_{j k} h(\tau) d \tau & \\
& \quad(1 \leq j<k \leq n),
\end{array}
$$

$$
\begin{aligned}
L_{0} \partial_{t} I_{\sigma}(t)= & L_{0} J_{\sigma}(t) \\
= & \sigma \cos \omega(t-\sigma) \cdot h(\sigma) \\
& \quad+\int_{\sigma}^{t} \cos \omega(t-\tau) \cdot h(\tau) d \tau \\
& \quad+\int_{\sigma}^{t} \cos \omega(t-\tau) \cdot L_{0} h(\tau) d \tau,
\end{aligned}
$$

$$
\begin{aligned}
L_{j} \partial_{k} I_{\sigma}(t)=\frac{\sin \omega(t-\sigma)}{\omega} \partial_{k}\left(x_{j} h(\sigma)\right) \\
\quad-\delta_{j k} \int_{\sigma}^{t} \cos \omega(t-\tau) \cdot h(\tau) d \tau \\
\quad+\int_{\sigma}^{t} \frac{\sin \omega(t-\tau)}{\omega} \partial_{k} L_{j} h(\tau) d \tau
\end{aligned}
$$

$$
(j, k=1, \ldots, n),
$$




$$
\begin{aligned}
\Omega_{j k} \partial_{\ell} I_{\sigma}(t)=\delta_{k \ell} \int_{\sigma}^{t} & \frac{\sin \omega(t-\tau)}{\omega} \partial_{j} h(\tau) d \tau \\
& -\delta_{j \ell} \int_{\sigma}^{t} \frac{\sin \omega(t-\tau)}{\omega} \partial_{k} h(\tau) d \tau \\
& +\int_{\sigma}^{t} \frac{\sin \omega(t-\tau)}{\omega} \partial_{\ell} \Omega_{j k} h(\tau) d \tau \\
L_{0} \partial_{k} I_{\sigma}(t)= & (1 \leq j<k \leq n, \quad \ell=1, \ldots, n), \\
& +\int_{\sigma}^{t} \frac{\sin \omega(t-\sigma)}{\omega} \partial_{k} h(\sigma) \\
& +\int_{\sigma}^{t} \frac{\sin \omega(t-\tau)}{\omega} \partial_{k} h(\tau) d \tau \\
&
\end{aligned}
$$

Proof. Throughout this proof $i$ means $\sqrt{-1}$. We start by showing (3.20). Taking the Fourier transform and carrying out a few integrations by part, we obtain

$$
\begin{gathered}
(3.26) \mathcal{F}\left[x_{j} \partial_{t} \int_{\sigma}^{t} \cos \omega(t-\tau) \cdot h(\tau) d \tau\right] \\
=i \partial_{\xi_{j}} \partial_{t} \int_{\sigma}^{t} \cos |\xi|(t-\tau) \cdot \hat{h}(\tau) d \tau \\
=\quad i \partial_{\xi_{j}}\{\cos |\xi|(t-\sigma) \cdot \hat{h}(\sigma)\}+i \partial_{\xi_{j}} \int_{\sigma}^{t} \cos |\xi|(t-\tau) \cdot \partial_{\tau} \hat{h}(\tau) d \tau \\
=\cos |\xi|(t-\sigma) \cdot i \partial_{\xi_{j}} \hat{h}(\sigma) \\
\quad+i \int_{\sigma}^{t}\left\{-\cos |\xi|(t-\tau) \cdot \xi_{j}(t-\tau) \hat{h}(\tau)-\sin |\xi|(t-\tau) \cdot \frac{\xi_{j}}{|\xi|} \hat{h}(\tau)\right\} d \tau \\
\quad+\int_{\sigma}^{t} \cos |\xi|(t-\tau) \cdot i \partial_{\xi_{j}} \partial_{\tau} \hat{h}(\tau) d \tau .
\end{gathered}
$$

On the other hand, we easily see that

$$
\begin{aligned}
t \partial_{j} J_{\sigma}(t)=\int_{\sigma}^{t} \cos \omega(t-\tau) \cdot( & t-\tau) \partial_{j} h(\tau) d \tau \\
& +\int_{\sigma}^{t} \cos \omega(t-\tau) \cdot \tau \partial_{j} h(\tau) d \tau
\end{aligned}
$$


Combining (3.26) and (3.27), we obtain (3.20). The proof of (3.21) is easy. Thus we omit it. We next show (3.22). Integrating by parts, we see that

$$
\begin{aligned}
& t \partial_{t} \int_{\sigma}^{t} \cos \omega(t-\tau) \cdot h(\tau) d \tau \\
=t \cos \omega(t-\sigma) \cdot h(\sigma)+\int_{\sigma}^{t} \cos \omega(t & -\tau) \cdot(t-\tau) \partial_{\tau} h(\tau) d \tau \\
& +\int_{\sigma}^{t} \cos \omega(t-\tau) \cdot \tau \partial_{\tau} h(\tau) d \tau .
\end{aligned}
$$

On the other hand, taking the Fourier transform, we get

$$
\begin{aligned}
& \mathcal{F}\left[x_{j} \partial_{j} \int_{\sigma}^{t} \cos \omega(t-\tau) \cdot h(\tau) d \tau\right] \\
&=-i \int_{\sigma}^{t} \sin |\xi|(t-\tau) \cdot \frac{\xi_{j}}{|\xi|}(t-\tau) i \xi_{j} \hat{h}(\tau) d \tau \\
&+\int_{\sigma}^{t} \cos |\xi|(t-\tau) \cdot i \partial_{\xi_{j}}\left\{i \xi_{j} \hat{h}(\tau)\right\} d \tau .
\end{aligned}
$$

Moreover, integration by parts yields

$$
\begin{aligned}
& -\sum_{j=1}^{n} i \int_{\sigma}^{t} \sin |\xi|(t-\tau) \cdot \frac{\xi_{j}}{|\xi|}(t-\tau) i \xi_{j} \hat{h}(\tau) d \tau \\
= & \int_{\sigma}^{t} \sin |\xi|(t-\tau) \cdot|\xi|(t-\tau) \hat{h}(\tau) d \tau \\
= & -\cos |\xi|(t-\sigma) \cdot(t-\sigma) \hat{h}(\sigma)+\int_{\sigma}^{t} \cos |\xi|(t-\tau) \cdot \hat{h}(\tau) d \tau \\
& -\int_{\sigma}^{t} \cos |\xi|(t-\tau) \cdot(t-\tau) \partial_{\tau} \hat{h}(\tau) d \tau .
\end{aligned}
$$

Combining (3.28)-(3.30), we obtain (3.22).

Next we prove (3.23)-(3.25). Repeating the same argument as in the proof of $(3.20)-(3.22)$, we get

$$
\begin{aligned}
L_{j} \partial_{k} I_{\sigma}(t)=\frac{\sin \omega(t-\sigma)}{\omega}\left(x_{j} \partial_{k} h(\sigma)\right) & \\
& +\int_{\sigma}^{t} \frac{\sin \omega(t-\tau)}{\omega} L_{j} \partial_{k} h(\tau) d \tau \\
&
\end{aligned}
$$




$$
\begin{aligned}
\Omega_{j k} \partial_{\ell} I_{\sigma}(t)= & \int_{\sigma}^{t} \frac{\sin \omega(t-\tau)}{\omega} \Omega_{j k} \partial_{\ell} h(\tau) d \tau \\
(1 \leq j<k \leq n, \ell=1, \ldots, n), & \\
L_{0} \partial_{k} I_{\sigma}(t)= & \frac{\sin \omega(t-\sigma)}{\omega} \partial_{k} h(\sigma)+2 \int_{\sigma}^{t} \frac{\sin \omega(t-\tau)}{\omega} \partial_{k} h(\tau) d \tau \\
+\int_{\sigma}^{t} \frac{\sin \omega(t-\tau)}{\omega} L_{0} \partial_{k} h(\tau) d \tau \quad & (k=1, \ldots, n) .
\end{aligned}
$$

Applying (3.2)-(3.4) to the right-hand sides of (3.31)-(3.33), we obtain (3.23)$(3.25)$.

Lemma 3.3. Suppose that $f \in \bigcap_{k=0}^{2} \dot{H}^{1+k, k}\left(\mathbf{R}^{n}\right), g \in \bigcap_{k=0}^{2} \dot{H}^{k, k}\left(\mathbf{R}^{n}\right)$. Set $\delta$ as in (2.1). Let $u_{0}^{-}=u_{0}^{-}(t, x)$ be a solution to $(1.3)$ with $\left(u_{0}^{-}(0), \partial_{t} u_{0}^{-}(0)\right)=$ $(f, g)$. Then $u_{0}^{-}$satisfies the following:

(3.35) $\partial_{t} u_{0}^{-} \in \bigcap_{k=0}^{2} C^{k}\left(\mathbf{R} ; H^{2-k}\left(\mathbf{R}^{n}\right)\right)$,

(3.36) $\Gamma^{\alpha} D_{k} u_{0}^{-} \in C\left(\mathbf{R} ; L^{2}\left(\mathbf{R}^{n}\right)\right)$ for any $\alpha$ with $|\alpha|=1,2$ and $k=0, \ldots, n$,

(3.37) $\sup _{t \in \mathbf{R}}\left\|D u_{0}^{-}(t)\right\|_{2,2} \leq C \delta$ with some constant $C>0$.

Proof. The following three equalities are necessary to prove this lemma:

$$
\begin{aligned}
L_{j} u_{0}^{-}(t) & =-\frac{\sin \omega t}{\omega} \partial_{j} f-\frac{\sin \omega t}{\omega} \omega^{2}\left(x_{j} f\right)+\cos \omega t \cdot\left(x_{j} g\right) \\
& =\frac{\sin \omega t}{\omega} \partial_{j} f+\frac{\sin \omega t}{\omega}\left[x_{j} \Delta f\right]+\cos \omega t \cdot\left(x_{j} g\right) \quad(j=1, \ldots, n), \\
\Omega_{j k} u_{0}^{-}(t) & =\cos \omega t \cdot\left(\Omega_{j k} f\right)+\frac{\sin \omega t}{\omega} \Omega_{j k} g \quad(1 \leq j<k \leq n) \\
L_{0} u_{0}^{-}(t) & =\sum_{j=1}^{n} \cos \omega t \cdot\left(x_{j} \partial_{j} f\right)-(n-1) \frac{\sin \omega t}{\omega} g+\sum_{j=1}^{n} \frac{\sin \omega t}{\omega} \partial_{j}\left(x_{j} g\right) .
\end{aligned}
$$

We may obtain these equalities easily by applying $L_{j}, \Omega_{j k}$ and $L_{0}$ to the formula

$$
u_{0}^{-}(t)=\cos \omega t \cdot f+\frac{\sin \omega t}{\omega} g .
$$


Then, taking care of (3.1) and with a simple equality $x_{j} \partial_{k} g=\partial_{k}\left(x_{j} g\right)-\delta_{j k} g$, we can prove this lemma without any difficulty. Thus, we omit the details of the proof.

Proposition 3.2. Let $n=2$, 3. Suppose that $h$ satisfies (3.17)-(3.19) and that $f \in \bigcap_{k=0}^{2} \dot{H}^{1+k, k}\left(\mathbf{R}^{n}\right), \quad g \in \bigcap_{k=0}^{2} \dot{H}^{k, k}\left(\mathbf{R}^{n}\right)$. Put $\delta$ as in (2.1). Then there exists a unique solution $u=u(t, x)$ to (3.15)-(3.16) satisfying

(3.38) $u \in C\left(\mathbf{R} ; \dot{H}^{1}\left(\mathbf{R}^{n}\right) \cap \dot{H}^{3}\left(\mathbf{R}^{n}\right)\right)$,

(3.39) $\partial_{t} u \in \bigcap_{k=0}^{2} C^{k}\left(\mathbf{R} ; H^{2-k}\left(\mathbf{R}^{n}\right)\right)$,

(3.40) $\Gamma^{\alpha} D_{k} u \in C\left(\mathbf{R} ; L^{2}\left(\mathbf{R}^{n}\right)\right)$ for any $\alpha$ with $|\alpha| \leq 2, k=0, \ldots, n$,

(3.41) $\sup _{t \in \mathbf{R}}\|D u(t)\|_{2,2} \leq C \delta+C \sup _{t \in \mathbf{R}}(1+|t|)^{(n-1)(\rho-1) / 2}\|h(t)\|_{2,2}$,

$$
\begin{array}{r}
\left\|D\left\{u(t)-u_{0}^{-}(t)\right\}\right\|_{2,2} \leq C(1-t)^{-(n-1)(\rho-1) / 2+1} \sup _{t \in \mathbf{R}}(1+|t|)^{(n-1)(\rho-1) / 2} \\
\times\|h(t)\|_{2,2} \text { for all } t<0 .
\end{array}
$$

Proof. Put

$$
u_{\sigma}(t):=u_{0}^{-}(t)+\int_{\sigma}^{t} \frac{\sin \omega(t-\tau)}{\omega} h(\tau) d \tau
$$

for any $\sigma \in(-\infty, 0)$.

In view of (3.17)-(3.19) and Lemma 3.3 it is easily verified that $u_{\sigma}$ is a unique solution to (3.15) satisfying

$$
\begin{aligned}
& u_{\sigma} \in C\left(\mathbf{R} ; \dot{H}^{1}\left(\mathbf{R}^{n}\right) \cap \dot{H}^{3}\left(\mathbf{R}^{n}\right)\right), \\
& \partial_{t} u_{\sigma} \in \bigcap_{k=0}^{2} C^{k}\left(\mathbf{R} ; H^{2-k}\left(\mathbf{R}^{n}\right)\right) .
\end{aligned}
$$

Moreover, Lemma 3.2 yields

$$
\begin{array}{r}
L_{j} \partial_{t} u_{\sigma}(t)=L_{j} \partial_{t} u_{0}^{-}(t)+\cos \omega(t-\sigma) \cdot\left(x_{j} h(\sigma)\right) \\
-\int_{\sigma}^{t} \frac{\sin \omega(t-\tau)}{\omega} \partial_{j} h(\tau) d \tau \\
+\int_{\sigma}^{t} \cos \omega(t-\tau) \cdot L_{j} h(\tau) d \tau
\end{array}
$$

$$
(j=1, \ldots, n),
$$




$$
\begin{aligned}
\Omega_{j k} \partial_{t} u_{\sigma}(t)=\Omega_{j k} \partial_{t} & u_{0}^{-}(t) \\
& +\int_{\sigma}^{t} \cos \omega(t-\tau) \cdot \Omega_{j k} h(\tau) d \tau
\end{aligned}
$$$$
(1 \leq j<k \leq n)
$$

$$
\begin{array}{r}
L_{0} \partial_{t} u_{\sigma}(t)=L_{0} \partial_{t} u_{0}^{-}(t)+\sigma \cos \omega(t-\sigma) \cdot h(\sigma) \\
+\int_{\sigma}^{t} \cos \omega(t-\tau) \cdot h(\tau) d \tau \\
+\int_{\sigma}^{t} \cos \omega(t-\tau) \cdot L_{0} h(\tau) d \tau \\
L_{j} \partial_{k} u_{\sigma}(t)=L_{j} \partial_{k} u_{0}^{-}(t)+\frac{\sin \omega(t-\sigma)}{\omega} \partial_{k}\left(x_{j} h(\sigma)\right) \\
\quad-\delta_{j k} \int_{\sigma}^{t} \cos \omega(t-\tau) \cdot h(\tau) d \tau \\
+\int_{\sigma}^{t} \frac{\sin \omega(t-\tau)}{\omega} \partial_{k} L_{j} h(\tau) d \tau
\end{array}
$$$$
(j, k=1, \ldots, n)
$$

(3.50) $\Omega_{j k} \partial_{\ell} u_{\sigma}(t)=\Omega_{j k} \partial_{\ell} u_{0}^{-}(t)+\delta_{k \ell} \int_{\sigma}^{t} \frac{\sin \omega(t-\tau)}{\omega} \partial_{j} h(\tau) d \tau$

$$
\begin{aligned}
& -\delta_{j \ell} \int_{\sigma}^{t} \frac{\sin \omega(t-\tau)}{\omega} \partial_{k} h(\tau) d \tau \\
& +\int_{\sigma}^{t} \frac{\sin \omega(t-\tau)}{\omega} \partial_{\ell} \Omega_{j k} h(\tau) d \tau \\
& \quad(1 \leq j<k \leq n, \ell=1, \ldots, n),
\end{aligned}
$$

$$
\begin{aligned}
L_{0} \partial_{k} u_{\sigma}(t)=L_{0} \partial_{k} & u_{0}^{-}(t)+\sigma \frac{\sin \omega(t-\sigma)}{\omega} \partial_{k} h(\sigma) \\
+ & \int_{\sigma}^{t} \frac{\sin \omega(t-\tau)}{\omega} \partial_{k} h(\tau) d \tau \\
& +\int_{\sigma}^{t} \frac{\sin \omega(t-\tau)}{\omega} \partial_{k} L_{0} h(\tau) d \tau \quad(k=1, \ldots, n) .
\end{aligned}
$$

Then it follows from (3.46)-(3.51) and (3.17)-(3.19) that

$$
\begin{aligned}
\Gamma^{\alpha} D_{k} u_{\sigma} \in \bigcap_{m=0}^{1} C^{m}\left(\mathbf{R} ; H^{1-m}\left(\mathbf{R}^{n}\right)\right) \\
\quad \text { for any } \alpha \text { with }|\alpha|=1 \text { and } k=0, \ldots, n .
\end{aligned}
$$


Since $h$ satisfies (3.17)-(3.19), we see that

$$
\begin{gathered}
u_{\sigma}(t) \rightarrow u(t):=u_{0}^{-}(t)+\int_{-\infty}^{t} \frac{\sin \omega(t-\tau)}{\omega} h(\tau) d \tau \\
\partial_{t} u_{\sigma}(t) \rightarrow z(t):=\partial_{t} u_{0}^{-}(t)+\int_{-\infty}^{t} \operatorname{in} C\left(\mathbf{R} ; \dot{H}^{1}\left(\mathbf{R}^{n}\right) \cap \dot{H}^{3}\left(\mathbf{R}^{n}\right)\right), \\
\cos \omega(t-\tau) \cdot h(\tau) d \tau \\
\operatorname{in~} \bigcap_{k=0}^{2} C^{k}\left(\mathbf{R} ; H^{2-k}\left(\mathbf{R}^{n}\right)\right)
\end{gathered}
$$

as $\sigma \rightarrow-\infty$. Clearly, $z=\partial_{t} u$, and $u$ is a solution to (3.15)-(3.16). Moreover, since

$$
\begin{aligned}
& \cos \omega(t-\sigma) \cdot\left(x_{j} h(\sigma)\right), \quad \sigma \cos \omega(t-\sigma) \cdot h(\sigma), \\
& \frac{\sin \omega(t-\sigma)}{\omega} \partial_{k}\left(x_{j} h(\sigma)\right), \quad \sigma \frac{\sin \omega(t-\sigma)}{\omega} \partial_{k} h(\sigma) \rightarrow 0 \\
& \text { in } \bigcap_{m=0}^{1} C^{m}\left(\mathbf{R} ; H^{1-m}\left(\mathbf{R}^{n}\right)\right) \text { as } \sigma \rightarrow-\infty
\end{aligned}
$$

by (3.17)-(3.19), it follows from (3.46)-(3.51) that there exists a function $u_{k \alpha}=$ $u_{k \alpha}(t, x)$ for every $k, \alpha$ such that

$$
\Gamma^{\alpha} D_{k} u_{\sigma} \rightarrow u_{k \alpha} \text { in } \bigcap_{m=0}^{1} C^{m}\left(\mathbf{R} ; H^{1-m}\left(\mathbf{R}^{n}\right)\right) \text { as } \sigma \rightarrow-\infty .
$$

To show $u_{k \alpha}=\Gamma^{\alpha} D_{k} u$ we proceed as follows. Taking account of (3.53), (3.54), we easily find that

$$
\int_{\mathbf{R}}\left(\Gamma^{\alpha} D_{k} u_{\sigma}(t), \varphi\right)_{L^{2}} \psi(t) d t \rightarrow \int_{\mathbf{R}}\left(\Gamma^{\alpha} D_{k} u(t), \varphi\right)_{L^{2}} \psi(t) d t
$$

as $\sigma \rightarrow-\infty$

for any $\varphi \in C_{0}^{\infty}\left(\mathbf{R}^{n}\right), \psi \in C_{0}^{\infty}(\mathbf{R})$. Here $(\cdot, \cdot)_{L^{2}}$ means the $L^{2}$-inner product. On the other hand, (3.56) implies that

$$
\int_{\mathbf{R}}\left(\Gamma^{\alpha} D_{k} u_{\sigma}(t), \varphi\right)_{L^{2}} \psi(t) d t \rightarrow \int_{\mathbf{R}}\left(u_{k \alpha}(t), \varphi\right)_{L^{2}} \psi(t) d t
$$

$$
\sigma \rightarrow-\infty
$$

for any $\varphi \in C_{0}^{\infty}\left(\mathbf{R}^{n}\right), \psi \in C_{0}^{\infty}(\mathbf{R})$. In view of (3.57), (3.58) we see that

$$
\left(\Gamma^{\alpha} D_{k} u(t)-u_{k \alpha}(t), \varphi\right)_{L^{2}}=0 \quad \text { a.e. } t \in \mathbf{R}
$$


for any $\varphi \in C_{0}^{\infty}\left(\mathbf{R}^{n}\right)$. It actually holds that $\left(u_{k \alpha}(t)-\Gamma^{\alpha} D_{k} u(t), \varphi\right)_{L^{2}}=0$ for arbitrary $t \in \mathbf{R}$ because $\left(u_{k \alpha}(t)-\Gamma^{\alpha} D_{k} u(t), \varphi\right)_{L^{2}} \in C(\mathbf{R})$. Hence it follows that $u_{k \alpha}(t)=\Gamma^{\alpha} D_{k} u(t)$ in $L^{2}\left(\mathbf{R}^{n}\right)$ for any $t \in \mathbf{R}$. Since

$$
u_{k \alpha} \in \bigcap_{m=0}^{1} C^{m}\left(\mathbf{R} ; H^{1-m}\left(\mathbf{R}^{n}\right)\right)
$$

by (3.56), we find that

$$
\Gamma^{\alpha} D_{k} u \in C\left(\mathbf{R} ; L^{2}\left(\mathbf{R}^{n}\right)\right)
$$

Repeating the same argument as in (3.57)-(3.59) for $D \Gamma^{\alpha} D_{k} u_{\sigma}$, we may conclude that

$$
\Gamma^{\alpha} D_{k} u \in \bigcap_{m=0}^{1} C^{m}\left(\mathbf{R} ; H^{1-m}\left(\mathbf{R}^{n}\right)\right) \text { for any } \alpha \text { with }|\alpha|=1 \text { and } k=0, \ldots, n \text {. }
$$

Moreover, recalling (3.46)-(3.51),(3.55), we obtain

$$
\begin{array}{r}
L_{j} \partial_{t} u(t)=L_{j} \partial_{t} u_{0}^{-}(t)-\int_{-\infty}^{t} \frac{\sin \omega(t-\tau)}{\omega} \partial_{j} h(\tau) d \tau \\
+\int_{-\infty}^{t} \cos \omega(t-\tau) \cdot L_{j} h(\tau) d \tau
\end{array}
$$

$$
(j=1, \ldots, n),
$$

$$
\Omega_{j k} \partial_{t} u(t)=\Omega_{j k} \partial_{t} u_{0}^{-}(t)+\int_{-\infty}^{t} \cos \omega(t-\tau) \cdot \Omega_{j k} h(\tau) d \tau
$$

$$
(1 \leq j<k \leq n)
$$

$$
\begin{aligned}
L_{0} \partial_{t} u(t)=L_{0} \partial_{t} & u_{0}^{-}(t)+\int_{-\infty}^{t} \cos \omega(t-\tau) \cdot h(\tau) d \tau \\
& +\int_{-\infty}^{t} \cos \omega(t-\tau) \cdot L_{0} h(\tau) d \tau
\end{aligned}
$$

$$
\begin{gathered}
L_{j} \partial_{k} u(t)=L_{j} \partial_{k} u_{0}^{-}(t)-\delta_{j k} \int_{-\infty}^{t} \cos \omega(t-\tau) \cdot h(\tau) d \tau \\
+\int_{-\infty}^{t} \frac{\sin \omega(t-\tau)}{\omega} \partial_{k} L_{j} h(\tau) d \tau
\end{gathered}
$$

$$
(j, k=1, \ldots, n),
$$




$$
\begin{aligned}
\Omega_{j k} \partial_{\ell} u(t)=\Omega_{j k} \partial_{\ell} u_{0}^{-}(t)+\delta_{k \ell} \int_{-\infty}^{t} \frac{\sin \omega(t-\tau)}{\omega} \partial_{j} h(\tau) d \tau & \\
& -\delta_{j \ell} \int_{-\infty}^{t} \frac{\sin \omega(t-\tau)}{\omega} \partial_{k} h(\tau) d \tau \\
& +\int_{-\infty}^{t} \frac{\sin \omega(t-\tau)}{\omega} \partial_{\ell} \Omega_{j k} h(\tau) d \tau \\
L_{0} \partial_{k} u(t)=L_{0} \partial_{k} u_{0}^{-}(t)+\int_{-\infty}^{t} \frac{\sin \omega(t-\tau)}{\omega} \partial_{k} h(\tau) d \tau & \\
& +\int_{-\infty}^{t} \frac{\sin \omega(t-\tau)}{\omega} \partial_{k} L_{0} h(\tau) d \tau \quad, \quad(k=1, \ldots, n) .
\end{aligned}
$$

In view of (3.17)-(3.19) it follows from (3.53)-(3.54) and (3.60)-(3.65) that

$$
\begin{aligned}
& \sup _{t \in \mathbf{R}}\|D u(t)\|_{1,2}+\sum_{|\alpha|=1} \sum_{k=0}^{n} \sup _{t \in \mathbf{R}}\left\|D \Gamma^{\alpha} D_{k} u(t)\right\|_{2} \\
\leq & C \delta+C \sup _{t \in \mathbf{R}}(1+|t|)^{(n-1)(\rho-1) / 2}\|h(t)\|_{2,2}
\end{aligned}
$$

and

$$
\begin{aligned}
& \left\|D\left\{u(t)-u_{0}^{-}(t)\right\}\right\|_{1,2}+\sum_{|\alpha|=1} \sum_{k=0}^{n}\left\|D \Gamma^{\alpha} D_{k}\left\{u(t)-u_{0}^{-}(t)\right\}\right\|_{2} \\
\leq & C(1-t)^{-(n-1)(\rho-1) / 2+1} \sup _{t \in \mathbf{R}}(1+|t|)^{(n-1)(\rho-1) / 2}\|h(t)\|_{2,2}
\end{aligned}
$$

for all $t<0$.

To complete the proof it remains to show

$$
\begin{aligned}
& \Gamma_{a} \Gamma_{b} D_{k} u \in C\left(\mathbf{R} ; L^{2}\left(\mathbf{R}^{n}\right)\right), \\
& \sup _{t \in \mathbf{R}}\left\|\Gamma_{a} \Gamma_{b} D_{k} u(t)\right\|_{2} \leq C \delta+C \sup _{t \in \mathbf{R}}(1+|t|)^{(n-1)(\rho-1) / 2}\|h(t)\|_{2,2}
\end{aligned}
$$

and

$$
\begin{aligned}
& \left\|\Gamma_{a} \Gamma_{b} D_{k}\left\{u(t)-u_{0}^{-}(t)\right\}\right\|_{2} \\
\leq & C(1-t)^{-(n-1)(\rho-1) / 2+1} \sup _{t \in \mathbf{R}}(1+|t|)^{(n-1)(\rho-1) / 2}\|h(t)\|_{2,2}
\end{aligned}
$$

for all $t<0$ 
for every $a, b=n+1, \ldots, \mu$ and $k=0, \ldots, n$. However, taking the assumptions on $h$ into account and making use of (3.60)-(3.65), we can show (3.68)-(3.70) only by repeating essentially the same argument. In fact, proceeding as before, we obtain

$$
\begin{aligned}
\Gamma_{a} \Gamma_{b} D_{k} u(t)=\Gamma_{a} \Gamma_{b} D_{k} u_{0}^{-}(t) & \\
& +\sum_{|\alpha| \leq 2} \sum_{j=1}^{n} C \int_{-\infty}^{t} \frac{\sin \omega(t-\tau)}{\omega} \partial_{j} \Gamma^{\alpha} h(\tau) d \tau \\
& +\sum_{|\alpha| \leq 2} \widetilde{C} \int_{-\infty}^{t} \cos \omega(t-\tau) \cdot \Gamma^{\alpha} h(\tau) d \tau
\end{aligned}
$$

with some constants $C, \widetilde{C}$ depending on $a, b, k, j, \alpha$ and $a, b, k, \alpha$, respectively. Then we can conclude that $\Gamma_{a} \Gamma_{b} D_{k} u$ satisfies (3.68)-(3.70).

Finally we show the uniqueness. Let $u_{1}$ and $u_{2}$ be solutions to (3.15)-(3.16). Since $u_{1}-u_{2}$ is a solution to (1.3), the energy conservation law for the free wave equation yields

$$
\left\|u_{1}(t)-u_{2}(t)\right\|_{e}=\left\|u_{1}(\sigma)-u_{2}(\sigma)\right\|_{e} \text { for any } t, \sigma \in \mathbf{R} .
$$

But it follows from (3.16) that $\left\|u_{1}(\sigma)-u_{2}(\sigma)\right\|_{e} \rightarrow 0$ as $\sigma \rightarrow-\infty$. Hence we can conclude that $\left\|u_{1}(t)-u_{2}(t)\right\|_{e}=0$ for any $t \in \mathbf{R}$. This implies uniqueness. Thus, the proof of Proposition 3.2 has been completed.

For the proof of Theorem 2.2 we need to consider the global solvability of the linear problem

$$
\begin{aligned}
& \square u=\partial_{t} h(t, x), \quad t \in \mathbf{R}, x \in \mathbf{R}^{3}, \\
& \left\|u(t)-u^{-}(t)\right\|_{e} \rightarrow 0 \quad(t \rightarrow-\infty) .
\end{aligned}
$$

Here $u^{-}$is a solution to (1.3) with $\left(u^{-}(0), \partial_{t} u^{-}(0)\right)=(f, g)$.

Lemma 3.4. Suppose that $f \in \bigcap_{k=0}^{2} \dot{H}^{k, k}\left(\mathbf{R}^{3}\right), \quad g \in \bigcap_{k=0}^{1} \dot{H}^{k, k+1}\left(\mathbf{R}^{3}\right)$. Set $\eta$ as in (2.12). Then $u^{-}$satisfies the following:

$$
\begin{aligned}
& u^{-} \in \bigcap_{k=0}^{2} C^{k}\left(\mathbf{R} ; H^{2-k}\left(\mathbf{R}^{3}\right)\right), \\
& \Gamma^{\alpha} u^{-} \in C\left(\mathbf{R} ; L^{2}\left(\mathbf{R}^{3}\right)\right) \text { for any } \alpha \text { with }|\alpha| \leq 2, \\
& \sup _{t \in \mathbf{R}}\left\|u^{-}(t)\right\|_{2,2} \leq C \eta .
\end{aligned}
$$


Proof. Taking care of Remark 2.1, we can prove this lemma in the same fashion as in the proof of Lemma 3.3. Thus, we omit the details.

Proposition 3.3. Let $n=3$. Suppose that $h$ satisfies

$$
\frac{1}{|\xi|} \hat{h} \in L^{\infty}\left(\mathbf{R} ; L^{2}\left(\mathbf{R}^{3}\right)\right) \text { and }\left\|\frac{1}{|\xi|} \hat{h}(t)\right\|_{2} \rightarrow 0 \text { as } t \rightarrow-\infty
$$

in addition to (3.17)-(3.19). Let $f \in \bigcap_{k=0}^{2} \dot{H}^{k, k}\left(\mathbf{R}^{3}\right), \quad g \in \bigcap_{k=0}^{1} \dot{H}^{k, k+1}\left(\mathbf{R}^{3}\right)$. Put $\eta$ as in (2.12). Then there exists a unique solution $u=u(t, x)$ to (3.72)(3.73) satisfying the following:

$$
\begin{aligned}
& u \in \bigcap_{k=0}^{2} C^{k}\left(\mathbf{R} ; H^{2-k}\left(\mathbf{R}^{3}\right)\right), \\
& \Gamma^{\alpha} u \in C\left(\mathbf{R} ; L^{2}\left(\mathbf{R}^{3}\right)\right) \text { for any } \alpha \text { with }|\alpha| \leq 2, \\
& \sup _{t \in \mathbf{R}}\|u(t)\|_{2,2} \leq C \eta+C \sup _{t \in \mathbf{R}}(1+|t|)^{\rho-1}\|h(t)\|_{2,2}, \\
& \left\|u(t)-u^{-}(t)\right\|_{2,2} \leq C(1-t)^{-\rho+2} \sup _{t \in \mathbf{R}}(1+|t|)^{\rho-1}\|h(t)\|_{2,2}
\end{aligned}
$$

for all $t<0$.

Proof. Put

$$
\begin{aligned}
u_{\sigma}(t):=u^{-}(t)+\int_{\sigma}^{t} \frac{\sin \omega(t-\tau)}{\omega} \partial_{\tau} h(\tau) d \tau & \\
& \text { for any } \sigma \in(-\infty, 0) .
\end{aligned}
$$

Carrying out integration by parts, we obtain

$$
\begin{aligned}
u_{\sigma}(t)=u^{-}(t) & -\frac{\sin \omega(t-\sigma)}{\omega} h(\sigma) \\
& +\int_{\sigma}^{t} \cos \omega(t-\tau) \cdot h(\tau) d \tau .
\end{aligned}
$$

In view of $(3.17)-(3.19),(3.77)$ we find from (3.83) that $u_{\sigma}$ is a unique solution to (3.72) satisfying

$$
u_{\sigma} \in \bigcap_{k=0}^{2} C^{k}\left(\mathbf{R} ; H^{2-k}\left(\mathbf{R}^{3}\right)\right) .
$$


Since it follows from $(3.19),(3.77)$ that

$$
\frac{\sin \omega(t-\sigma)}{\omega} h(\sigma) \rightarrow 0 \text { in } \bigcap_{k=0}^{2} C^{k}\left(\mathbf{R} ; H^{2-k}\left(\mathbf{R}^{3}\right)\right)
$$

as $\sigma \rightarrow-\infty$, we see that

$$
\begin{aligned}
u_{\sigma}(t) \rightarrow u(t):=u^{-}(t)+\int_{-\infty}^{t} \cos \omega(t-\tau) \cdot h(\tau) d \tau & \\
& \operatorname{in} \bigcap_{k=0}^{2} C^{k}\left(\mathbf{R} ; H^{2-k}\left(\mathbf{R}^{3}\right)\right)
\end{aligned}
$$

as $\sigma \rightarrow-\infty$. Moreover, (3.85) implies that $u$ is a solution to (3.72) satisfying

$$
\begin{aligned}
\text { (3.86) } \sum_{k=0}^{2} \sup _{t \in \mathbf{R}}\left\|\partial_{t}^{k} u(t)\right\|_{H^{2-k}} & \leq C \eta+C \sup _{t \in \mathbf{R}}(1+|t|)^{\rho-1}\|h(t)\|_{2,2}, \\
\text { (3.87) } \sum_{k=0}^{2}\left\|\partial_{t}^{k}\left\{u(t)-u^{-}(t)\right\}\right\|_{H^{2-k}} \leq & C(1-t)^{-\rho+2} \\
& \cdot \sup _{t \in \mathbf{R}}(1+|t|)^{\rho-1}\|h(t)\|_{2,2} \text { for all } t<0 .
\end{aligned}
$$

To complete the proof it remains to show that $u$ in (3.85) satisfies

$$
\begin{array}{ll}
\text { (3.88) } & \Gamma_{a} u, \Gamma_{a} \Gamma_{b} u \in C\left(\mathbf{R} ; L^{2}\left(\mathbf{R}^{3}\right)\right), \\
(3.89) \quad & \sup _{t \in \mathbf{R}}\left\|\Gamma_{a} u(t)\right\|_{2}, \sup _{t \in \mathbf{R}}\left\|\Gamma_{a} \Gamma_{b} u(t)\right\|_{2} \leq C \eta+C \sup _{t \in \mathbf{R}}(1+|t|)^{\rho-1}\|h(t)\|_{2,2}, \\
(3.90) \quad & \left\|\Gamma_{a}\left\{u(t)-u^{-}(t)\right\}\right\|_{2},\left\|\Gamma_{a} \Gamma_{b}\left\{u(t)-u^{-}(t)\right\}\right\|_{2} \\
\leq & C(1-t)^{-\rho+2} \sup _{t \in \mathbf{R}}(1+|t|)^{\rho-1}\|h(t)\|_{2,2} \quad \text { for all } t<0
\end{array}
$$

for every $a, b=4, \ldots, 10$. Put

$$
u^{\sigma}(t):=u^{-}(t)+\int_{\sigma}^{t} \cos \omega(t-\tau) \cdot h(\tau) d \tau \quad \text { for any } \sigma \in(-\infty, 0)
$$

Taking account of the assumptions on $h$, we can show in quite the same way as in the proof of Proposition 3.2 that the problem (3.72)-(3.73) has a unique global solution $u$ satisfying (3.88)-(3.90) and 
(3.91) $\Gamma^{\alpha} u(t)=\Gamma^{\alpha} u^{-}(t)+\sum_{|\beta| \leq|\alpha|} C \int_{-\infty}^{t} \cos \omega(t-\tau) \cdot \Gamma^{\beta} h(\tau) d \tau$

$$
+\sum_{j=1}^{3} \sum_{|\beta| \leq|\alpha|-1} \widetilde{C} \int_{-\infty}^{t} \frac{\sin \omega(t-\tau)}{\omega} \partial_{j} \Gamma^{\beta} h(\tau) d \tau
$$

for every $\alpha$ with $|\alpha|=1,2$.

Here $C$ and $\widetilde{C}$ are constants depending on $\alpha, \beta$ and $\alpha, \beta, j$, respectively. Hence, we omit the details.

4. Proof of Theorem 2.1. We introduce the set $X_{E}$ of functions as follows:

$$
\begin{aligned}
X_{E}=\{u=u(t, x) \mid & u \in C\left(\mathbf{R} ; \dot{H}^{1}\left(\mathbf{R}^{n}\right) \cap \dot{H}^{3}\left(\mathbf{R}^{n}\right)\right), \\
& \partial_{t} u \in \bigcap_{k=0}^{2} C^{k}\left(\mathbf{R} ; H^{2-k}\left(\mathbf{R}^{n}\right)\right), \\
& \Gamma^{\alpha} D_{k} u \in C\left(\mathbf{R} ; L^{2}\left(\mathbf{R}^{n}\right)\right) \text { for }|\alpha|=1,2 \text { and } \\
& \left.k=0,1, \ldots, n,\|u\|:=\sup _{t \in \mathbf{R}}\|D u(t)\|_{2,2} \leq E\right\}
\end{aligned}
$$

with $E>0$. Endowed with the metric $d(u, v):=\|u-v\|\left(u, v \in X_{E}\right), X_{E}$ is a complete metric space for any $E>0$. We construct a solution to (1.1) in $X_{2 C_{1} \delta}$ with some $C_{1}>0$ by a modified iteration argument as in John [7].

The following proposition is an immediate consequence of Lemma 3.3.

Proposition 4.1. Suppose that $f \in \bigcap_{k=0}^{2} \dot{H}^{1+k, k}\left(\mathbf{R}^{n}\right), g \in \bigcap_{k=0}^{2} \dot{H}^{k, k}\left(\mathbf{R}^{n}\right)$. Put $\delta$ as in (2.1). Let $u_{0}^{-}$be a solution to (1.3) with $\left(u_{0}^{-}(0), \partial_{t} u_{0}^{-}(0)\right)=(f, g)$. Then,

$$
u_{0}^{-} \in X_{C_{1} \delta} \text { with some constant } C_{1}>0 .
$$

Lemma 4.1. $F(D u)$ has the following properties for any $u \in X_{E}$ with $|D u| \leq$ 1 (cf. (3.17)-(3.19)) :

$$
\begin{aligned}
& \Gamma^{\alpha} F(D u) \in C\left(\mathbf{R} ; L^{2}\left(\mathbf{R}^{n}\right)\right), \\
& \left\||x| \Gamma^{\alpha} F(D u(t))\right\|_{2} \leq C E^{\rho}(1+|t|)^{-(n-1)(\rho-1) / 2+1}
\end{aligned}
$$

for any $\alpha$ with $|\alpha| \leq 1$ and

$$
\|F(D u(t))\|_{2,2} \leq C E^{\rho}(1+|t|)^{-(n-1)(\rho-1) / 2} .
$$


Proof. We begin by showing (4.4). Taking (F.2) into account and making use of Klainerman's inequality (3.7), we see that

$$
\begin{aligned}
\|F(D u(t))\|_{1,2} & \leq C(1+|t|)^{-(n-1)(\rho-1) / 2}\|D u(t)\|_{2,2}^{\rho-1}\|D u(t)\|_{1,2} \\
& \leq C E^{\rho}(1+|t|)^{-(n-1)(\rho-1) / 2} .
\end{aligned}
$$

Moreover, in view of the chain rule and applying (3.7) with $s=2$ and (3.9) with $s=1, p=2, q=4$, we obtain for $|\alpha|=2$

$$
\begin{aligned}
\left\|\Gamma^{\alpha} F(D u(t))\right\|_{2} \leq & C(1+|t|)^{-(n-1)(\rho-1) / 2}\|D u(t)\|_{2,2}^{\rho} \\
& +C(1+|t|)^{-(n-1)(\rho-2) / 2}\|D u(t)\|_{2,2}^{\rho-2}\|D u(t)\|_{1,4}^{2} \\
\leq & C(1+|t|)^{-(n-1)(\rho-1) / 2}\|D u(t)\|_{2,2}^{\rho} \\
\leq & C E^{\rho}(1+|t|)^{-(n-1)(\rho-1) / 2} .
\end{aligned}
$$

Combining (4.5) and (4.6) leads us to (4.4). Let us next show (4.3). Applying Klainerman's inequality (3.7) again, we find that

$$
\begin{aligned}
& \sum_{|\alpha| \leq 1}\left\||x| \Gamma^{\alpha} F(D u(t))\right\|_{2}^{2} \\
& \leq C \sum_{|\alpha| \leq 1} \sum_{k=0}^{n} \int_{\mathbf{R}^{n}} \frac{|x|^{2}}{(1+|t|+|x|)^{(n-1)(\rho-1)}}\left|\Gamma^{\alpha} D_{k} u(t, x)\right|^{2} d x \\
& \leq C(1+|t|)^{-(n-1)(\rho-1)+2} E^{2 \rho} . \\
& \leq\|D u(t)\|_{2,2}^{2(\rho-1)}
\end{aligned}
$$

This is just (4.3). Finally, we verify (4.2). However, (4.2) follows immediately from (F.2) and (3.5). Thus, we omit the computational details.

For any given $v \in X_{E}$ with $|D v| \leq 1$ let us consider the global solvability of the problem

$$
\begin{aligned}
& \square u=F(D v), \quad t \in \mathbf{R}, x \in \mathbf{R}^{n}, \\
& \left\|u(t)-u_{0}^{-}(t)\right\|_{e} \rightarrow 0 \quad(t \rightarrow-\infty) .
\end{aligned}
$$

Noting (4.1) and Lemma 4.1, we find from Proposition 3.2 that the problem (4.8)-(4.9) has a unique solution $u=u(t, x)$ satisfying

(4.10) $u \in C\left(\mathbf{R} ; \dot{H}^{1}\left(\mathbf{R}^{n}\right) \cap \dot{H}^{3}\left(\mathbf{R}^{n}\right)\right)$,

$$
\partial_{t} u \in \bigcap_{k=0}^{2} C^{k}\left(\mathbf{R} ; H^{2-k}\left(\mathbf{R}^{n}\right)\right),
$$

(4.12) $\quad \Gamma^{\alpha} D_{k} u \in C\left(\mathbf{R} ; L^{2}\left(\mathbf{R}^{n}\right)\right)$ for any $\alpha$ with $|\alpha| \leq 2$ and $k=0, \ldots, n$,

(4.13) $\sup _{t \in \mathbf{R}}\|D u(t)\|_{2,2} \leq C_{1} \delta+C_{4} E^{\rho}$ with some $C_{1}, C_{4}>0$,

$$
\left\|D\left\{u(t)-u_{0}^{-}(t)\right\}\right\|_{2,2} \leq C E^{\rho}(1-t)^{-(n-1)(\rho-1) / 2+1} \text { for all } t<0 .
$$


Taking $\delta$ small so that

$$
2 C_{1} \delta C_{3} \leq 1 \text { and } C_{4}\left(2 C_{1} \delta\right)^{\rho} \leq C_{1} \delta
$$

may hold, we can then define a sequence $\left\{u_{m}\right\} \subset X_{2 C_{1} \delta}$ inductively by solving the problem

$$
\begin{aligned}
& \square u_{m+1}=F\left(D u_{m}\right), \quad t \in \mathbf{R}, x \in \mathbf{R}^{n}, \\
& \left\|u_{m+1}(t)-u_{0}^{-}(t)\right\|_{e} \rightarrow 0 \quad(t \rightarrow-\infty)
\end{aligned}
$$

for $m=0,1, \ldots$ and $u_{0}=u_{0}^{-}$. Employing a modified iteration argument as in John [7], we will show that $\left\{u_{m}\right\}$ is a Cauchy sequence in $X_{2 C_{1} \delta}$.

Proposition 4.2. The sequence $\left\{u_{m}\right\}$ defined by (4.16)-(4.17) satisfies the following estimates with some constants $C_{j}(j=5, \ldots, 8)$ :

$$
\begin{gathered}
\text { (4.18) }\left\|u_{m+1}-u_{m}\right\| \leq \quad C_{5}\left(\left\|u_{m}\right\|+\left\|u_{m-1}\right\|\right)^{\rho-1}\left\|u_{m}-u_{m-1}\right\| \quad \text { if } n=2, \\
\begin{aligned}
(4.19)\left\|u_{m+1}-u_{m}\right\| \leq & C_{6}\left(\left\|u_{m}\right\|+\left\|u_{m-1}\right\|\right)^{2(\rho-2) / 3+2} \\
& \times\left\{\sup _{t \in \mathbf{R}}\left\|D u_{m}(t)-D u_{m-1}(t)\right\|_{2}\right\}^{(\rho-2) / 3} \\
& +C_{7}\left(\left\|u_{m}\right\|+\left\|u_{m-1}\right\|\right)^{\rho-1}\left\|u_{m}-u_{m-1}\right\| \\
\text { if } n=3,2<\rho<3, & \\
(4.20)\left\|u_{m+1}-u_{m}\right\| \leq & C_{8}\left(\left\|u_{m}\right\|+\left\|u_{m-1}\right\|\right)^{\rho-1}\left\|u_{m}-u_{m-1}\right\| \quad \text { if } n=3, \rho \geq 3 .
\end{aligned}
\end{gathered}
$$

Proof. For simplicity, we put $u_{m+1}^{*}=u_{m+1}-u_{m}, u_{m}^{*}=u_{m}-u_{m-1}$. We have only to show (4.19) because the others follow with much ease. Since

$$
u_{m+1}(t)=u_{0}^{-}(t)+\int_{-\infty}^{t} \frac{\sin \omega(t-\tau)}{\omega} F\left(D u_{m}(\tau)\right) d \tau
$$

by (3.53), we get, as in (4.5),

$$
\begin{aligned}
\left\|D u_{m+1}^{*}(t)\right\|_{2} \leq & C \int_{-\infty}^{t}\left\|F\left(D u_{m}(\tau)\right)-F\left(D u_{m-1}(\tau)\right)\right\|_{2} d \tau \\
\leq & C \int_{-\infty}^{t}(1+|\tau|)^{-(\rho-1)} d \tau\left(\sup _{t \in \mathbf{R}}\left\|D u_{m}(t)\right\|_{2,2}\right. \\
& \left.\quad+\sup _{t \in \mathbf{R}}\left\|D u_{m-1}(t)\right\|_{2,2}\right)^{\rho-1} \sup _{t \in \mathbf{R}}\left\|D u_{m}^{*}(t)\right\|_{2}
\end{aligned}
$$




$$
\leq C\left(\left\|u_{m}\right\|+\left\|u_{m-1}\right\|\right)^{\rho-1} \sup _{t \in \mathbf{R}}\left\|D u_{m}^{*}(t)\right\|_{2}
$$

We next estimate $\Gamma^{\alpha} D_{k} u_{m+1}^{*}$ for $|\alpha|=1,2$ and $k=0, \ldots, n$. Taking account of (3.60)-(3.65) and applying (3.9) with $p=2, q=4$ and $s=1$ as well as Klainerman's inequality (3.7), we see that

$$
\begin{gathered}
\sum_{|\alpha|=1} \sum_{k=0}^{n}\left\|\Gamma^{\alpha} D_{k} u_{m+1}^{*}(t)\right\|_{2} \\
\leq C \int_{-\infty}^{t}\left(\frac{\left\|D u_{m}(\tau)\right\|_{2,2}+\left\|D u_{m-1}(\tau)\right\|_{2,2}}{1+|\tau|}\right)^{\rho-2} \\
\quad \times\left\|D u_{m}^{*}(\tau)\right\|_{4}\left\|D u_{m}(\tau)\right\|_{1,4} d \tau \\
\quad+C \int_{-\infty}^{t}\left(\frac{\left\|D u_{m-1}(\tau)\right\|_{2,2}}{1+|\tau|}\right)^{\rho-1}\left\|D u_{m}^{*}(\tau)\right\|_{1,2} d \tau \\
\leq C\left(\left\|u_{m}\right\|+\left\|u_{m-1}\right\|\right)^{\rho-1} \sup _{t \in \mathbf{R}}\left\|D u_{m}^{*}(t)\right\|_{1,2} \cdot
\end{gathered}
$$

Moreover, by virtue of (3.71), we have

$$
\begin{aligned}
& \sum_{|\alpha|=2} \sum_{k=0}^{n}\left\|\Gamma^{\alpha} D_{k} u_{m+1}^{*}(t)\right\|_{2} \\
& \leq C \sum_{|\alpha| \leq 1} \int_{-\infty}^{t}\left\|\Gamma^{\alpha} F\left(D u_{m}(\tau)\right)-\Gamma^{\alpha} F\left(D u_{m-1}(\tau)\right)\right\|_{2} d \tau \\
& \quad+C \sum_{|\alpha|=2} \int_{-\infty}^{t}\left\|\Gamma^{\alpha} F\left(D u_{m}(\tau)\right)-\Gamma^{\alpha} F\left(D u_{m-1}(\tau)\right)\right\|_{2} d \tau .
\end{aligned}
$$

The estimate for the first term on the right-hand side of (4.23) can be done in quite the same way as before. However, in the case $n=3$ with $2<\rho<3$ the estimate for the second term becomes complicated because of the lack of smoothness in $F$. Set

$$
\frac{\rho-2}{3}+\varepsilon^{-1}=1 \text {. }
$$

Using Klainerman's inequality (3.7) and Hölder's inequality together with this one,

$$
\left|u_{m}^{*}\right|^{\rho-2} \leq\left(\left|u_{m}\right|+\left|u_{m-1}\right|\right)^{2(\rho-2) / 3}\left|u_{m}^{*}\right|^{(\rho-2) / 3},
$$

which is elementary, we see that

$$
\sum_{|\alpha|=2}\left\|\Gamma^{\alpha} F\left(D u_{m}(\tau)\right)-\Gamma^{\alpha} F\left(D u_{m-1}(\tau)\right)\right\|_{2} \leq
$$




$$
\begin{aligned}
& \leq\left(\frac{\left\|D u_{m}(\tau)\right\|_{2,2}+\left\|D u_{m-1}(\tau)\right\|_{2,2}}{1+|\tau|}\right)^{2(\rho-2) / 3} \\
& \quad \times\left\|D u_{m}^{*}(\tau)\right\|_{2}^{(\rho-2) / 3}\left\|D u_{m}(\tau)\right\|_{1,4 \varepsilon}^{2} \\
& \quad+\left(\frac{\left\|D u_{m-1}(\tau)\right\|_{2,2}}{1+|\tau|}\right)^{\rho-2}\left\|D u_{m}^{*}(\tau)\right\|_{1,4}\left\|D u_{m}(\tau)\right\|_{1,4} \\
& \quad+\left(\frac{\left\|D u_{m}(\tau)\right\|_{2,2}+\left\|D u_{m-1}(\tau)\right\|_{2,2}}{1+|\tau|}\right)^{\rho-2} \\
& \quad+\frac{\left\|D u_{m}^{*}(\tau)\right\|_{2,2}}{1+|\tau|}\left\|D u_{m}(\tau)\right\|_{2,2} \\
& \quad+\left(\frac{\left\|D u_{m-1}(\tau)\right\|_{2,2}}{1+|\tau|}\right)^{\rho-1}\left\|D u_{m}^{*}(\tau)\right\|_{2,2} .
\end{aligned}
$$

Note that a simple calculation shows $1 /(4 \varepsilon)>1 / 6$ for any $\rho$ with $2<\rho<3$. Then, applying (3.9) with $p=2, q=4 \varepsilon$ and $s=1$ to the first term on the right-hand side of (4.24), we obtain

$$
\begin{aligned}
& \sum_{|\alpha|=2}\left\|\Gamma^{\alpha} F\left(D u_{m}(\tau)\right)-\Gamma^{\alpha} F\left(D u_{m-1}(\tau)\right)\right\|_{2} \\
& \leq C\left[( 1 + | \tau | ) ^ { - 2 ( \rho - 2 ) / 3 - 2 \{ 1 / 2 - 1 / ( 4 \varepsilon ) \} \times 2 } \left(\left\|u_{m}\right\|\right.\right. \\
& \left.\quad+\left\|u_{m-1}\right\|\right)^{2(\rho-2) / 3+2} \\
& \quad \times \sup _{\tau \in \mathbf{R}}\left\|D u_{m}^{*}(\tau)\right\|_{2}^{(\rho-2) / 3} \\
& \quad+(1+|\tau|)^{-(\rho-2)-2(1 / 2-1 / 4) \times 2}\left(\left\|u_{m}\right\|+\left\|u_{m-1}\right\|\right)^{\rho-1}\left\|u_{m}^{*}\right\| \\
& \left.\quad+(1+|\tau|)^{-(\rho-1)}\left(\left\|u_{m}\right\|+\left\|u_{m-1}\right\|\right)^{\rho-1}\left\|u_{m}^{*}\right\|\right] .
\end{aligned}
$$

A simple observation shows $-2(\rho-2) / 3-4\{1 / 2-1 /(4 \varepsilon)\}=-(\rho-1)<-1$, which together with (4.21)-(4.25) leads to the estimate (4.19).

Take $\delta$ still smaller so that

$$
2 C_{1} \delta \leq \min \left\{\frac{1}{2}, \frac{1}{C_{3}}\right\} \quad \text { and } \quad C_{9}\left(4 C_{1} \delta\right)^{\rho-1} \leq \frac{1}{2}
$$

may hold, where $C_{9}=\max \left\{C_{j} \mid 4 \leq j \leq 8\right\}$. Now we are ready to show that $\left\{u_{m}\right\}$ is a Cauchy sequence in $X_{2 C_{1} \delta}$. It is enough to treat the case where $n=3$ and $2<\rho<3$. In view of (4.26) it follows from (4.21) that

$$
\begin{aligned}
\sup _{t \in \mathbf{R}}\left\|D u_{m+1}^{*}(t)\right\|_{2} & \leq \frac{1}{2} \sup _{t \in \mathbf{R}}\left\|D u_{m}^{*}(t)\right\|_{2} \\
& \leq\left(\frac{1}{2}\right)^{m} \sup _{t \in \mathbf{R}}\left\|D u_{1}^{*}(t)\right\|_{2} \leq 4 C_{1} \delta\left(\frac{1}{2}\right)^{m} .
\end{aligned}
$$


Substituting (4.27) into (4.19), we get

$$
\begin{aligned}
\left\|u_{m+1}^{*}\right\| & \leq C_{6}\left(4 C_{1} \delta\right)^{2(\rho-2) / 3+2}\left\{4 C_{1} \delta\left(\frac{1}{2}\right)^{m-1}\right\}^{(\rho-2) / 3}+\frac{1}{2}\left\|u_{m}^{*}\right\| \\
& \leq\left(\frac{1}{2}\right)^{(\rho-2)(m-1) / 3+1}+\frac{1}{2}\left\|u_{m}^{*}\right\| \\
& \leq\left(\frac{1}{2}\right)^{(\rho-2)(m-1) / 3+1}+\frac{1}{2}\left(\frac{1}{2}\right)^{(\rho-2)(m-2) / 3+1} \\
& \leq 2\left(\frac{1}{2}\right)^{(\rho-2)(m-1) / 3+1}+\left(\frac{1}{2}\right)^{2}\left\|u_{m-1}^{*}\right\| \\
& \leq \cdots \\
& \leq m\left(\frac{1}{2}\right)^{(\rho-2)(m-1) / 3+1}+\left(\frac{1}{2}\right)^{m}\left\|u_{m-1}^{*}\right\| .
\end{aligned}
$$

Here we have used $1 / 2 \leq(1 / 2)^{(\rho-2) / 3}$ repeatedly. Since

$$
\sum_{m=1}^{\infty} m\left(\frac{1}{2}\right)^{(\rho-2)(m-1) / 3+1}<\infty,
$$

$\left\{u_{m}\right\}$ is a Cauchy sequence in $X_{2 C_{1} \delta}$. As we have mentioned at the beginning of this section, $X_{2 C_{1} \delta}$ is complete with respect to the metric $d\left(v, v^{\prime}\right)=\left\|v-v^{\prime}\right\|$, $\left\{u_{m}\right\}$ thus converges uniformly with respect to $t$ as $m \rightarrow \infty$ toward a certain $u \in X_{2 C_{1} \delta}$, which is a solution to (1.1) with the properties (2.2)-(2.6).

Finally, uniqueness follows from (4.15), (4.19) and (4.21) in the case where $n=3$ and $2<\rho<3$ or from (4.15), (4.18) and (4.20) in the other cases.

We next prove the part (ii) of Theorem 2.1. Define

$$
u_{0}^{+}(t):=u(t)+\int_{t}^{\infty} \frac{\sin \omega(t-\tau)}{\omega} F(D u(\tau)) d \tau
$$

or equivalently

$$
u_{0}^{+}(t)=u_{0}^{-}(t)+\int_{-\infty}^{\infty} \frac{\sin \omega(t-\tau)}{\omega} F(D u(\tau)) d \tau
$$

for the solution $u$ to (1.1) obtained above. Then, proceeding as before, we can verify that $u_{0}^{+}$is a solution to (1.3) with the properties (2.7)-(2.11). Uniqueness of the solutions to (1.3) satisfying (2.7)-(2.11) easily follows from the energy conservation law for solutions to (1.3). Thus, we have completed the proof of Theorem 2.1. 
5. Proof of Theorem 2.2. We denote by $Z_{E}$ the following set of functions:

$$
\begin{aligned}
Z_{E}=\{u=u(t, x) \mid & u \in \bigcap_{k=0}^{2} C^{k}\left(\mathbf{R} ; H^{2-k}\left(\mathbf{R}^{3}\right)\right), \\
& \Gamma^{\alpha} u \in C\left(\mathbf{R} ; L^{2}\left(\mathbf{R}^{3}\right)\right) \text { for any } \alpha \text { with }|\alpha| \leq 2, \\
& \left.\|u\|_{Z}:=\sup _{t \in \mathbf{R}}\|u(t)\|_{2,2} \leq E\right\}
\end{aligned}
$$

for $E>0$. Endowed with the metric $d_{Z}(u, v):=\|u-v\|_{Z}, Z_{E}$ is a complete metric space for any $E$. We seek a solution to (1.2) in $Z_{2 C_{2} \eta}$ with some $C_{2}>0$ by following the argument in Section 4 .

Lemma 3.4 yields

Proposition 5.1. Suppose that $f \in \bigcap_{k=0}^{2} \dot{H}^{k, k}\left(\mathbf{R}^{3}\right), g \in \bigcap_{k=0}^{1} \dot{H}^{k, k+1}\left(\mathbf{R}^{3}\right)$. Put $\eta$ as in (2.12). Let $u^{-}$be a solution to (1.3) with $\left(u^{-}(0), \partial_{t} u^{-}(0)\right)=(f, g)$. Then:

$$
u^{-} \in Z_{C_{2} \eta} \text { with some constant } C_{2}>0 .
$$

Lemma 5.1. $G(u)$ has the following properties for any $u \in Z_{E}$ with $|u| \leq 1$ (cf. (3.17)-(3.19), (3.77)) :

$$
\begin{aligned}
& \Gamma^{\alpha} G(u) \in C\left(\mathbf{R} ; L^{2}\left(\mathbf{R}^{3}\right)\right), \\
& \left\||x| \Gamma^{\alpha} G(u(t))\right\|_{2} \leq C E^{\rho}(1+|t|)^{-\rho+2}
\end{aligned}
$$

for any $\alpha$ with $|\alpha| \leq 1$ and

$$
\begin{aligned}
& \|G(u(t))\|_{2,2} \leq C E^{\rho}(1+|t|)^{-\rho+1}, \\
& \frac{1}{|\xi|} \mathcal{F}[G(u)] \in L^{\infty}\left(\mathbf{R} ; L^{2}\left(\mathbf{R}^{3}\right)\right), \\
& \left\|\frac{1}{|\xi|} \mathcal{F}[G(u(t))]\right\|_{2} \rightarrow 0 \quad \text { as } t \rightarrow-\infty .
\end{aligned}
$$

Proof. The proofs for (5.2)-(5.4) are quite the same as those for (4.2)(4.4). Therefore we have only to show (5.5). Since $\widehat{u * v}=\widehat{u v}$ for $u \in \mathcal{S}^{\prime}\left(\mathbf{R}^{n}\right)$, $v \in \mathcal{S}\left(\mathbf{R}^{n}\right)$ and $\mathcal{F}\left[|x|^{-2}\right]=a|\xi|^{-1}$ with some constant $a$ when $n=3$, we obtain by applying (3.10) first and then (3.7), (3.9)

$$
\begin{aligned}
\| \frac{1}{|\xi|} \mathcal{F}[ & G(u(t))]\left\|_{2} \leq C\right\||u(t)|^{\rho} \|_{6 / 5} \\
& \leq C \sup _{x \in \mathbf{R}^{3}}|u(t, x)|^{\rho-2}\|u(t)\|_{3}\|u(t)\|_{2} \\
& \leq C(1+|t|)^{-\rho+2-2(1 / 2-1 / 3)}\|u(t)\|_{2,2}^{\rho-2}\|u(t)\|_{1,2}\|u(t)\|_{2} \\
& \leq C(1+|t|)^{-\rho+5 / 3} E^{\rho} .
\end{aligned}
$$


This implies (5.5).

Remark 5.1. Note that $\mathcal{F}\left[|x|^{-1}\right]=a|\xi|^{-1}$ with some constant $a$ when $n=2$. Since we encounter the case $p=1$ in (3.10), we were not able to discuss the scattering theory for (1.2) with $n=2$ in the same way.

Remark 5.2. We have derived the first inequality in (5.6) rather formally. However, this can be justified by the density of $\mathcal{S}\left(\mathbf{R}^{3}\right)$ in $L^{6 / 5}\left(\mathbf{R}^{3}\right)$, the HausdorffYoung inequality (see, e.g., [5] on page 165) and weak sequential compactness of closed balls in $L^{2}\left(\mathbf{R}^{3}\right)$.

For any given $v \in Z_{E}$ with $|v| \leq 1$ let us consider the global solvability of the linear problem

$$
\begin{aligned}
& \square u=\partial_{t} G(v), \quad t \in \mathbf{R}, x \in \mathbf{R}^{3}, \\
& \left\|u(t)-u^{-}(t)\right\|_{e} \rightarrow 0 \quad(t \rightarrow-\infty) .
\end{aligned}
$$

Noting (5.1) and Lemma 5.1, we find by applying Proposition 3.3 that the problem (5.7)-(5.8) has a unique solution $u=u(t, x)$ satisfying

$$
\begin{aligned}
& u \in \bigcap_{k=0}^{2} C^{k}\left(\mathbf{R} ; H^{2-k}\left(\mathbf{R}^{3}\right)\right), \\
& \Gamma^{\alpha} u \in C\left(\mathbf{R} ; L^{2}\left(\mathbf{R}^{3}\right)\right) \text { for any } \alpha \text { with }|\alpha| \leq 2, \\
& \sup _{t \in \mathbf{R}}\|u(t)\|_{2,2} \leq C_{2} \eta+C_{10} E^{\rho} \text { with some } C_{2}, C_{10}>0, \\
& \left\|u(t)-u^{-}(t)\right\|_{2,2} \leq C E^{\rho}(1-t)^{-\rho+2} \text { for all } t<0 .
\end{aligned}
$$

Recalling (3.85), (3.91), we can prove Theorem 2.2 only by repeating quite the same argument as in the proof of Theorem 2.1. Thus, we omit the remaining part of the proof.

6. Final remarks. We can apply our arguments to construct global solutions to the Cauchy problem for (1.1) or (1.2) with small initial data given at a finite time.

Consider the Cauchy problem

$$
\left\{\begin{array}{l}
\square u=F(D u), \quad t \geq 0, x \in \mathbf{R}^{n}, \\
u(0)=f, \partial_{t} u(0)=g .
\end{array}\right.
$$

For $(f, g) \in\left(\bigcap_{k=0}^{2} \dot{H}^{1+k, k}\left(\mathbf{R}^{n}\right)\right) \times\left(\bigcap_{k=0}^{2} \dot{H}^{k, k}\left(\mathbf{R}^{n}\right)\right)$, we introduce the set $\widetilde{X}_{E}$ 
of functions

$$
\begin{aligned}
\widetilde{X}_{E}=\{u=u(t, x) \mid & u \in C\left([0, \infty) ; \dot{H}^{1}\left(\mathbf{R}^{n}\right) \cap \dot{H}^{3}\left(\mathbf{R}^{n}\right)\right) \\
& \partial_{t} u \in \bigcap_{k=0}^{2} C^{k}\left([0, \infty) ; H^{2-k}\left(\mathbf{R}^{n}\right)\right), \\
& \Gamma^{\alpha} D_{k} u \in C\left([0, \infty) ; L^{2}\left(\mathbf{R}^{n}\right)\right) \text { for }|\alpha|=1,2 \text { and } \\
& k=0, \ldots, n, u(0)=f, \partial_{t} u(0)=g \\
& \left.\|u\|_{\widetilde{X}}:=\sup _{t \geq 0}\|D u(t)\|_{2,2} \leq E\right\}
\end{aligned}
$$

with $E>0$. For $(f, g)$, put $\delta$ as in $(2.1)$. Endowed with the metric $d_{\widetilde{X}}(u, v):=$ $\|u-v\|_{\widetilde{X}}, \widetilde{X}_{E}$ is a nonempty complete metric space if $\delta$ is sufficiently small relative to $E$. Repeating the same argument as in the proof of Theorem 2.1, we obtain the following result.

Theorem 6.1. Let $n=2,3$. Suppose that (F.1)-(F.3) hold and that $f \in$ $\cap_{k=0}^{2} \dot{H}^{1+k, k}\left(\mathbf{R}^{n}\right), g \in \cap_{k=0}^{2} \dot{H}^{k, k}\left(\mathbf{R}^{n}\right)$. Then there exists a positive constant $\delta_{1}$ depending on $n, \rho, A_{1}$ and $A_{2}$ such that if $\delta \leq \delta_{1}$, then the problem (6.1) has a unique solution $u$ in $\widetilde{X}_{C \delta}$ with some constant $C>0$.

Remark 6.1. We have succeeded in removing the assumption of spherical symmetry on initial data from Theorem 1 in Sideris [14], where it was shown that when $n=3$ and $F=F\left(\partial_{t} u,|\nabla u|\right)$, (6.1) has a unique global radial solution provided that $f$ and $g$ are small and spherically symmetric.

Next let us consider the Cauchy problem

$$
\left\{\begin{array}{l}
\square u=\partial_{t} G(u), \quad t \geq 0, x \in \mathbf{R}^{3}, \\
u(0)=f, \partial_{t} u(0)=g .
\end{array}\right.
$$

For $(f, g) \in\left(\bigcap_{k=0}^{2} \dot{H}^{k, k}\right) \times\left(\bigcap_{k=0}^{1} \dot{H}^{k, k+1}\right)$, we introduce the set $\widetilde{Z}_{E}$ of functions

$$
\begin{aligned}
\widetilde{Z}_{E}=\{u=u(t, x) \mid & u \in \bigcap_{k=0}^{2} C^{k}\left([0, \infty) ; H^{2-k}\left(\mathbf{R}^{3}\right)\right) \\
& \Gamma^{\alpha} u \in C\left([0, \infty) ; L^{2}\left(\mathbf{R}^{3}\right)\right) \text { for any } \alpha \text { with } \\
& |\alpha| \leq 2, u(0)=f, \partial_{t} u(0)=g, \\
& \left.\|u\|_{\tilde{Z}}=\sup _{t \geq 0}\|u(t)\|_{2,2} \leq E\right\}
\end{aligned}
$$

Endowed with the metric $d_{\widetilde{Z}}=\|u-v\|_{\widetilde{Z}}, \widetilde{Z}_{E}$ is a nonempty complete metric space if $\eta$ is sufficiently small relative to $E$. Repeating the same argument as in the proof of Theorem 2.2, we obtain 
Theorem 6.2. Let $n=3$. Suppose that (G.1)-(G.3) hold and that $f \in$ $\cap_{k=0}^{2} \dot{H}^{k, k}\left(\mathbf{R}^{3}\right), g \in \cap_{k=0}^{1} \dot{H}^{k, k+1}\left(\mathbf{R}^{3}\right)$. Then there exists a positive constant $\eta_{1}$ depending on $\eta, A_{3}$ and $A_{4}$ such that if $\eta \leq \eta_{1}$, then the problem (6.2) has a unique solution $u$ in $\widetilde{Z}_{C \eta}$ with some $C>0$.

Remark 6.2. All the conclusions of Theorem 6.2 are valid for the Cauchy problem for (2.21) in $\mathbf{R}^{+} \times \mathbf{R}^{3}$ with initial data $\left(u(0), \partial_{t} u(0)\right)=(f, g)$ if we replace $\eta_{1}$ by a suitable positive number.

Acknowledgements. The second author would like to thank Professors Yoshio Tsutsumi and Tohru Ozawa for their valuable advice.

Added in proof. Recently, N. Tzvetkov ("Existence of global solutions to nonlinear massless Dirac system and wave equation with small data," Preprint) has independently obtained the same result as our Theorem 6.1 , by using a similar method.

\section{REFERENCES}

[1] R. A. Adams, Sobolev Spaces, Academic Press, New York et al., 1975.

[2] R. AGemi, Blow-up of solutions to nonlinear wave equations in two space dimensions, Manuscripta Math. 73 (1991), 153-162.

[3] R. T. GLassey, Blow-up theorems for nonlinear wave equations, Math. Z. 132 (1973), 183-203.

[4] P. Godin, Lifespan of solutions of semilinear wave equations in two space dimensions, Comm. P. D. E. 18 (1993), 895-916.

[5] L. Hörmander, The analysis of linear partial differential operators 1, SpringerVerlag, Berlin et al., 1983.

[6] L. Hörmander, On Sobolev spaces associated with some Lie algebras, Current Topics in P. D. E., Kinokuniya, Japan, 1986, 261-287.

[7] F. John, Blow-up of solutions of nonlinear wave equations in three space dimensions, Manuscripta Math. 28 (1979), 235-268.

[8] F. John, Blow-up of solutions for quasi-linear wave equations in three space dimensions, Comm. Pure Appl. Math. 34 (1981), 29-51.

[9] S. Klainerman, Uniform decay estimates and the Lorentz invariance of the classical wave equations, Comm. Pure Appl. Math. 38 (1985), 321-332.

[10] S. Klainerman, Remarks on the global Sobolev inequalities in the Minkowski space $\mathbf{R}^{n+1}$, Comm. Pure Appl. Math. 40 (1987), 111-117.

[11] J. L. Lions \& W. A. Strauss, Some non-linear evolution equations, Bull. Soc. Math. France. 93 (1965), 43-96.

[12] K. MASUDA, Blow-up of solutions for quasi-linear wave equations in two space dimensions, Lecture Notes in Num. Appl. Anal. 6, North-Holland, Kinokuniya, Tokyo et al., 1983, 87-91.

[13] J. Schaeffer, Finite-time blow-up for $u_{t t}-\Delta u=H\left(u_{r}, u_{t}\right)$ in two space dimensions, Comm. P. D. E. 11 (1986), 513-543.

[14] T. C. SidERIS, Global behavior of solutions to nonlinear wave equations in three dimensions, Comm. P. D. E. 8 (1983), 1291-1323. 
[15] T. C. SiDERIS, Formation of singularities in solutions to nonlinear hyperbolic equations, Arch. Rat. Mech. Anal. 86 (1984), 369-381.

[16] K. YoshidA, Functional Analysis, the 6th ed., Springer-Verlag, Berlin et al., 1980.

Kunio Hidano

Department of Mathematics

School of Science and Engineering

Waseda University

Tokyo 169, Japan

Kimitoshi Tsutaya

Department of Mathematics

Hokkaido University

Sapporo 060, Japan

Received: March 24th, 1995. 